\title{
Well-posedness for fractional Navier-Stokes equations in critical spaces close to $\dot{B}_{\infty, \infty}^{-(2 \beta-1)}\left(\mathbb{R}^{n}\right)$
}

\author{
Zhichun Zhai
}

Communicated by Jack Xin, received November 21, 2009.

\begin{abstract}
In this paper, we study the well-posedness for the fractional NavierStokes equations in critical spaces $G_{n}^{-(2 \beta-1)}\left(\mathbb{R}^{n}\right)$ and $B M O^{-(2 \beta-1)}\left(\mathbb{R}^{n}\right)$ which are close to the largest critical space $\dot{B}_{\infty, \infty}^{-(2 \beta-1)}\left(\mathbb{R}^{n}\right)$. In $G_{n}^{-(2 \beta-1)}\left(\mathbb{R}^{n}\right)$, we establish the well-posedness based on a priori estimates for the fractional Navier-Stokes equations in Besov spaces. To obtain the well-posedness in $B M O^{-(2 \beta-1)}\left(\mathbb{R}^{n}\right)$, we find a relationship between $Q_{\alpha ; \infty}^{\beta,-1}\left(\mathbb{R}^{n}\right)$ and $B M O\left(\mathbb{R}^{n}\right)$ by giving an equivalent characterization of $B M O^{-\zeta}\left(\mathbb{R}^{n}\right)$. As an application, we get the well-posedness for fractional magnetohydrodynamics equations in $G_{n}^{-(2 \beta-1)}\left(\mathbb{R}^{n}\right)$ and $B M O^{-(2 \beta-1)}\left(\mathbb{R}^{n}\right)$.
\end{abstract}

\section{Contents}

1. Introduction

2. Preliminary lemmas 31

3. Proof of main results 34

References

\section{Introduction}

In this paper, we study the well-posedness of mild solutions to the fractional Navier-Stokes equations on the half-space $\mathbb{R}_{+}^{1+n}=(0, \infty) \times \mathbb{R}^{n}, n \geq 2$ :

$$
\begin{cases}\partial_{t} u+(-\triangle)^{\beta} u+(u \cdot \nabla) u-\nabla p=0, & \text { in } \mathbb{R}_{+}^{1+n} \\ \nabla \cdot u=0, & \text { in } \mathbb{R}_{+}^{1+n} \\ \left.u\right|_{t=0}=a, & \text { in } \mathbb{R}^{n}\end{cases}
$$

2000 Mathematics Subject Classification. Primary 35Q30; 76D03; 42B35; 46E30.

Key words and phrases. Navier-Stokes equations; Magnetohydrodynamics equations; $B M O^{-\zeta}\left(\mathbb{R}^{n}\right) ;$ Besov spaces.

Project supported in part by Natural Science and Engineering Research Council of Canada.

(C)2010 International Press 
with $\beta \in(1 / 2,1)$. The mild solution to equations (1.1) is the fixed point of operator

$$
(T u)(t, x)=e^{-t(-\triangle)^{\beta}} a(x)-\int_{0}^{t} e^{-(t-s)(-\triangle)^{\beta}} P \nabla(u \otimes u)(s, x) d s .
$$

Here

$$
e^{-t(-\triangle)^{\beta}} f(x):=K_{t}^{\beta}(x) * f(x) \quad \text { with } \quad \widehat{K_{t}^{\beta}}(\xi)=e^{-t|\xi|^{2 \beta}}
$$

and $P$ is the Helmboltz-Weyl projection:

$$
P=\left\{P_{j, k}\right\}_{j, k=1, \cdots, n}=\left\{\delta_{j, k}+R_{j} R_{k}\right\}_{j, k=1, \cdots, n}
$$

with the Kronecker symbol $\delta_{j, k}$ and the Riesz transform $R_{j}=\partial_{j}(-\triangle)^{-1 / 2}$.

Note that the following scaling

$$
u_{\lambda}(t, x)=\lambda^{2 \beta-1} u\left(\lambda^{2 \beta} t, \lambda x\right), \quad p_{\lambda}(t, x)=\lambda^{4 \beta-2} p\left(\lambda^{2 \beta} t, \lambda x\right), \quad a_{\lambda}(x)=\lambda^{2 \beta-1} a(\lambda x)
$$

is important for equations (1.1). This leads us to study equations (1.1) in critical function spaces which are invariant under the scaling $f(x) \longrightarrow \lambda^{2 \beta-1} f(\lambda x)$.

When $\beta=1$, equations (1.1) become the classical Navier-Stokes equations. The existence of mild solutions has been established locally in time and global for small initial data in various critical spaces. Especially, Koch and Tataru in [13] proved the well-posedness of classical Navier-Stokes equations in the space $B M O^{-1}\left(\mathbb{R}^{n}\right)=\nabla \cdot\left(B M O\left(\mathbb{R}^{n}\right)\right)^{n}$. Xiao in $[\mathbf{2 4}]$ generalized the results of Koch and Tataru [13] to $Q_{\alpha ; \infty}^{-1}\left(\mathbb{R}^{n}\right)$ for $\alpha \in(0,1)$. Chen and Xin in [5] studied the classical Navier-Stokes equations in several critical spaces. See, Kato [12], Cannone [3], Giga and Miyakawa [9], Bourgain and Pavlović [2] and the references therein for more history and recent development.

For general case, Lions $[\mathbf{1 6}]$ proved the global existence of the classical solutions to equations (1.1) when $\beta \geq \frac{5}{4}$ in dimensional 3. Wu in [19] obtained a similar result for $\beta \geq \frac{1}{2}+\frac{n}{4}$ in dimension $n$. For the important case $\beta<\frac{1}{2}+\frac{n}{4}$, Wu in $[\mathbf{2 0}, \mathbf{2 1}]$ considered the existence of solution to equations $(1.1)$ in $\dot{B}_{p, q}^{1+\frac{n}{p}-2 \beta}\left(\mathbb{R}^{n}\right)$. In Li and Zhai $[\mathbf{1 4}, \mathbf{1 5}]$, inspired by Koch and Tataru $[\mathbf{1 3}]$ and Xiao [24], they studied equations (1.1) in critical space $Q_{\alpha ; \infty}^{\beta,-1}\left(\mathbb{R}^{n}\right)=\nabla \cdot\left(Q_{\alpha}^{\beta}\left(\mathbb{R}^{n}\right)\right)^{n}$ for $\beta \in(1 / 2,1)$ and $\alpha \in(0, \beta)$. Here $Q_{\alpha}^{\beta}\left(\mathbb{R}^{n}\right)$ for $\alpha \in(-\infty, \beta)$ is the set of all measurable functions with

$$
\sup _{I}(l(I))^{2(\alpha+\beta-1)-n} \int_{I} \int_{I} \frac{|f(x)-f(y)|^{2}}{|x-y|^{n+2(\alpha-\beta+1)}} d x d y<\infty
$$

where the supremum is taken over all cubes $I$ with the edge length $l(I)$ and the edges parallel to the coordinate axes in $\mathbb{R}^{n} \cdot Q_{\alpha}^{\beta}\left(\mathbb{R}^{n}\right)$ is a generalization of $Q_{\alpha}\left(\mathbb{R}^{n}\right)$ studied by Essen, Janson, Peng and Xiao [7], Xiao [23], Dafni and Xiao [6] and reference therein. Meanwhile, Li and Zhai $[\mathbf{1 4}]$ proved that Besov space $\dot{B}_{\infty, \infty}^{1-2 \beta}\left(\mathbb{R}^{n}\right)$ for $\beta \in(1 / 2,1)$ is the biggest one among the critical spaces of equations (1.1).

In this paper, we accomplish two major goals. First, we prove the wellposedness for equations $(1.1)$ in spaces $G_{n}^{-(2 \beta-1)}\left(\mathbb{R}^{n}\right)$ for $\beta \in(1 / 2,1)$. Here, for $s>0$,

$$
\begin{aligned}
G_{p}^{-s}\left(\mathbb{R}^{n}\right)= & \left\{f \in \mathcal{S}^{\prime}\left(\mathbb{R}^{n}\right):|f| \in \mathcal{S}^{\prime}\left(\mathbb{R}^{n}\right),\right. \\
& \left.\|f\|_{G_{p}^{-s}\left(\mathbb{R}^{n}\right)}=\sup _{t>0} t^{\frac{s n}{2 p \beta}}\left\|e^{-t(-\triangle)^{\beta}}|f|\right\|_{L^{\infty}\left(\mathbb{R}^{n}\right)}<\infty\right\}
\end{aligned}
$$


which is a subclass of Besov spaces and also contains the Morrey-type space of measures. Second, to obtain the well-posedness in $B M O^{-(2 \beta-1)}\left(\mathbb{R}^{n}\right)$ for $\beta \in(1 / 2,1)$, we find a relation between $Q_{\alpha, \infty}^{\beta,-1}\left(\mathbb{R}^{n}\right)$ and $B M O\left(\mathbb{R}^{n}\right)$ :

$$
Q_{\alpha, \infty}^{\beta,-1}\left(\mathbb{R}^{n}\right)=(-\triangle)^{\frac{2 \beta-1}{2}} B M O\left(\mathbb{R}^{n}\right)=B M O^{-(2 \beta-1)}\left(\mathbb{R}^{n}\right)
$$

for $\alpha=1-\beta, \beta \in(1 / 2,1)$, by giving an equivalent characterization of $B M O^{-\zeta}\left(\mathbb{R}^{n}\right)$. Our well-posedness results extend that of Chen and Xin [5], Koch and Tataru [13]. The relation (1.3) between $Q_{\alpha, \infty}^{\beta,-1}\left(\mathbb{R}^{n}\right)$ for $\beta \in(1 / 2,1)$ and $B M O\left(\mathbb{R}^{n}\right)$ gives us a clear link between $Q_{\alpha}^{\beta}\left(\mathbb{R}^{n}\right)$ and $B M O\left(\mathbb{R}^{n}\right)$. When $\alpha \neq 1-\beta$, an interesting problem is whether or not there is a similar link between $Q_{\alpha}^{\beta}\left(\mathbb{R}^{n}\right)$ and $B M O\left(\mathbb{R}^{n}\right)$. We do not know the answer now. As an application, we study the well-posedness for fractional magnetohydrodynamics equations in critical spaces $G_{n}^{-(2 \beta-1)}\left(\mathbb{R}^{n}\right)$ and $B M O^{-(2 \beta-1)}\left(\mathbb{R}^{n}\right)$.

The space $B M O^{-\zeta}\left(\mathbb{R}^{n}\right)$ was introduced by Zhou and Gala by using heat semigroup $e^{t \triangle}$. In the following, we define $B M O^{-\zeta}\left(\mathbb{R}^{n}\right)$ by $e^{-t(-\triangle)^{\beta}}$ for $\beta \in(1 / 2,1)$. This is motivated by the following well-known facts.

For a $C^{\infty}$ real-valued function on $\mathbb{R}^{n}$ satisfying the properties:

$$
\begin{gathered}
\phi_{j} \in L^{1}\left(\mathbb{R}^{n}\right),\left|\phi_{j}(x)\right| \lesssim(1+|x|)^{-(n+1)}, \\
\int_{\mathbb{R}^{n}} \phi_{j}(x) d x=0 \text { and }\left(\phi_{j}\right)_{t}(x)=t^{-n} \phi_{j}\left(\frac{x}{t}\right), \\
f \in B M O\left(\mathbb{R}^{n}\right) \Longleftrightarrow \sup _{x \in \mathbb{R}^{n}, r \in(0, \infty)} r^{-n} \int_{0}^{r} \int_{|y-x|<r}\left|f * \phi_{t}(y)\right|^{2} t^{-1} d t d y<\infty .
\end{gathered}
$$

Here $A \lesssim B$ means $A \leq C B$ with $C>0$. Thus $B M O\left(\mathbb{R}^{n}\right)$ can be defined equivalently as

$$
\|f\|_{B M O\left(\mathbb{R}^{n}\right)}^{2}=\sup _{x \in \mathbb{R}^{n}, r \in(0, \infty)} r^{-n} \int_{0}^{r^{2 \beta}} \int_{|y-x|<r}\left|\nabla e^{-t(-\triangle)^{\beta}} f(y)\right|^{2} t^{\frac{1-\beta}{\beta}} d t d y<\infty .
$$

Then, (1.6) leads us to introduce $B M O^{-\zeta}\left(\mathbb{R}^{n}\right)$ as follows.

Definition 1.1. For $\beta \in(1 / 2,1), 0 \leq \zeta \leq n / 2$, define $B M O^{-\zeta}\left(\mathbb{R}^{n}\right)$ as the set of all measurable functions $f$ with

$$
\|f\|_{B M O^{-\zeta\left(\mathbb{R}^{n}\right)}}^{2}=\sup _{x \in \mathbb{R}^{n}, r \in(0, \infty)} r^{-n} \int_{0}^{r^{2 \beta}} \int_{|y-x|<r} t^{\frac{\zeta-\beta}{\beta}}\left|e^{-t(-\triangle)^{\beta}} f(y)\right|^{2} d t d y<\infty .
$$

Obviously, $B M O^{-\zeta}\left(\mathbb{R}^{n}\right)$ is invariant under the scaling $f(x) \longrightarrow \lambda^{\zeta} f(\lambda x)$. Note that $Q_{\alpha ; \infty}^{\beta,-1}\left(\mathbb{R}^{n}\right)$ is invariant under the scaling $f(x) \longrightarrow \lambda^{2 \beta-1} f(\lambda x)$. Thus $B M O^{-\zeta}\left(\mathbb{R}^{n}\right)$ will be more useful than $Q_{\alpha ; \infty}^{\beta,-1}\left(\mathbb{R}^{n}\right)$.

We state our main results as follows. The first one is a priori estimates in homogeneous Besov spaces for the fractional Navier-Stokes equations.

Propisition 1.2. Let $2-2 \beta<w<2 \beta, 1+n / p+w<4 \beta, 2 \leq n \leq p \leq \infty$, $1 \leq q \leq \infty$ and

$$
a \in\left(\mathcal{S}^{\prime}\left(\mathbb{R}^{n}\right)\right)^{n}, \quad f(t) \in\left(\dot{B}_{p, \infty}^{w-2 \beta+\frac{n}{p}}\left(\mathbb{R}^{n}\right)\right)^{n \times n} .
$$


Then the solution to the integral equation

$$
u(t)=e^{-t(-\triangle)^{\beta}} a+\int_{0}^{t} e^{-(t-s)(-\triangle)^{\beta}} P \nabla \cdot f(s) d s
$$

satisfies the estimates

$$
\|u(t)\|_{\dot{B}_{p, q}^{-1+\frac{n}{p}}\left(\mathbb{R}^{n}\right)} \lesssim\|a\|_{\dot{B}_{p, q}^{-(2 \beta-1)+\frac{n}{p}}\left(\mathbb{R}^{n}\right)}+\sup _{0<s<t} s^{\frac{w}{2 \beta}+1-\frac{1}{\beta}}\|f(s)\|_{\dot{B}_{p, \infty}^{w-2 \beta+\frac{n}{p}}}\left(\mathbb{R}^{n}\right)
$$

and

$$
t^{\frac{w}{2 \beta}}\|u(t)\|_{\dot{B}_{p, \infty}^{w-(2 \beta-1)+\frac{n}{p}}\left(\mathbb{R}^{n}\right)} \lesssim\|a\|_{\dot{B}_{p, \infty}^{-(2 \beta-1)+\frac{n}{p}}\left(\mathbb{R}^{n}\right)}+\sup _{0<s<t} s^{\frac{w}{2 \beta}}\|f(s)\|_{\dot{B}_{p, \infty}^{w-2 \beta+\frac{n}{p}}}\left(\mathbb{R}^{n}\right)
$$

provided the right-hand sides of the above inequalities are finite, respectively.

Applying Proposition 1.2, we obtain the existence of solution to equations (1.1).

Theorem 1.3. Let $n \geq 2, \beta \in(1 / 2,1), \max \{2 \beta-1,2-2 \beta\}<w<2 \beta$, $1+n / p+w<4 \beta, a \in G_{n}^{-(2 \beta-1)}\left(\mathbb{R}^{n}\right), \nabla \cdot a=0$. If $\|a\|_{G_{n}^{-(2 \beta-1)}\left(\mathbb{R}^{n}\right)}$ is small enough, then there is a unique solution to (1.1) satisfying

$$
\|u(t)\|_{G_{n}^{-(2 \beta-1)}\left(\mathbb{R}^{n}\right)}+t^{\frac{1}{2 \beta}}\|u(t)\|_{L^{\infty}\left(\mathbb{R}^{n}\right)}+t^{\frac{w}{2 \beta}}\|u(t)\|_{\dot{B}_{\infty, \infty}^{w-(2 \beta-1)}\left(\mathbb{R}^{n}\right)} \lesssim\|a\|_{G_{n}^{-(2 \beta-1)}\left(\mathbb{R}^{n}\right)} .
$$

Here it is worth particularly mentioning that our previous result gives us the existence of solution to equations (1.1) with initial data in Morrey-type spaces of Radon measures. In fact, for $0<s \leq p<\infty$, one has

$$
M_{p / s}\left(\mathbb{R}^{n}\right)=\left\{f \in G_{p}^{-s}\left(\mathbb{R}^{n}\right): f \quad \text { is a locally finte Radon measure }\right\} .
$$

Here $M_{q}\left(\mathbb{R}^{n}\right)(1 \leq q \leq \infty)$ is the space of distributions $f \in \mathcal{S}^{\prime}\left(\mathbb{R}^{n}\right)$ such that $f$ is a locally finite Radon measure with

$$
\|f\|_{M_{q}\left(\mathbb{R}^{n}\right)}:=\sup _{x \in \mathbb{R}^{n}, t>0} t^{\frac{n}{q}-n}|f|(B(x, t))<\infty
$$

where $|f|$ is the total variation of $f$ and $B(x, t)$ is the open ball in $\mathbb{R}^{n}$ with radius $t$ centered at $x$.

By Proposition 1.2, we get the existence of solutions to equations (1.1) in $\dot{B}_{p, \infty}^{-(2 \beta-1)+\frac{n}{p}}\left(\mathbb{R}^{n}\right)$.

Propisition 1.4. Let $n \geq 2, \beta \in(1 / 2,1), n \leq p<\infty, \max \{2 \beta-n / p, 2-2 \beta\}<$ $w<2 \beta$ and $1+n / p+w<4 \beta$. Assume that $a \in\left(\dot{B}_{p, \infty}^{-(2 \beta-1)+\frac{n}{p}}\left(\mathbb{R}^{n}\right)\right)^{n}$ and $\nabla \cdot a=0$. If $\|a\|_{\dot{B}_{p, \infty}^{-(2 \beta-1)+\frac{n}{p}}\left(\mathbb{R}^{n}\right)}$ is small enough, then there exists a unique solution to equations (1.1) satisfying

$$
\begin{aligned}
& \|u(t)\|_{\dot{B}_{p, \infty}^{-(2 \beta-1)+\frac{n}{p}}\left(\mathbb{R}^{n}\right)}+t^{\frac{2 \beta-1}{2 \beta}}\|u(t)\|_{L^{\infty}\left(\mathbb{R}^{n}\right)}+t^{\frac{w}{2 \beta}}\|u(t)\|_{\dot{B}_{p, \infty}^{w-(2 \beta-1)+\frac{n}{p}}\left(\mathbb{R}^{n}\right)} \\
& \lesssim\|a\|_{\dot{B}_{p, \infty}^{-(2 \beta-1)+\frac{n}{p}}\left(\mathbb{R}^{n}\right)} .
\end{aligned}
$$

In [21], Wu established a result similar to Proposition 1.4 by using lower bounds for the integral involving $(-\triangle)^{\beta}$.

Now, we study the properties of $B M O^{-\zeta}\left(\mathbb{R}^{n}\right)$. 
Theorem 1.5. (BMO $\mathrm{B}^{-\zeta}$ and Besov spaces) Let $\beta \in(1 / 2,1)$. For any $f \in$ $\mathcal{S}^{\prime}\left(\mathbb{R}^{n}\right)$ and $r>0$, one has

$r^{\zeta}\left\|e^{-r^{2 \beta}(-\triangle)^{\beta}} f\right\|_{L^{\infty}\left(\mathbb{R}^{n}\right)} \lesssim\left(r^{-n} \int_{0}^{r^{2 \beta}} \int_{\left|x-x_{0}\right| \leq r} s^{\zeta-1+\frac{1-\beta}{\beta}}\left|e^{-s(-\triangle)^{\beta}} f(x)\right|^{2} d x d s\right)^{1 / 2}$, that is, $B M O^{-\zeta}\left(\mathbb{R}^{n}\right) \hookrightarrow \dot{B}_{\infty, \infty}^{-\zeta}\left(\mathbb{R}^{n}\right)$.

THEOREM 1.6. A distribution $f$ belongs to $B M O^{-\zeta}\left(\mathbb{R}^{n}\right)$ if and only if there exists a distribution $g \in B M O\left(\mathbb{R}^{n}\right)$ such that $f=(-\triangle)^{\frac{\zeta}{2}} g$.

Zhou and Gala established results similar to Theorems 1.5-1.6 for $B M O^{-\zeta}\left(\mathbb{R}^{n}\right)$ defined by heat semigroup $e^{t \Delta}$. Thus, $B M O^{-\zeta}\left(\mathbb{R}^{n}\right)$ is independent of $e^{-t(-\triangle)^{\beta}}$ for $\beta \in(1 / 2,1]$.

It follows from the definition of $B M O^{-\zeta}\left(\mathbb{R}^{n}\right)$ and $Q_{\alpha ; \infty}^{\beta,-1}\left(\mathbb{R}^{n}\right)$ (see [14]) that when $\alpha=1-\beta, Q_{\alpha ; \infty}^{\beta,-1}\left(\mathbb{R}^{n}\right)=B M O^{-\zeta}\left(\mathbb{R}^{n}\right)$ for $\zeta=2 \beta-1$. Thus, we can obtain the existence of mild solution to equations (1.1) with initial data in $B M O^{-(2 \beta-1)}\left(\mathbb{R}^{n}\right)$ as follows.

We need to define some notations.

Definition 1.7. Let $1 / 2<\beta<1$.

(i) A tempered distribution $f$ on $\mathbb{R}^{n}$ belongs to $B M O_{T}^{-(2 \beta-1)}\left(\mathbb{R}^{n}\right)$ provided

$$
\begin{aligned}
& \|f\|_{B M O_{T}^{-(2 \beta-1)}\left(\mathbb{R}^{n}\right)}= \\
& \sup _{x \in \mathbb{R}^{n}, r \in(0, T)}\left(r^{-n} \int_{0}^{r^{2 \beta}} \int_{|y-x|<r}\left|K_{t}^{\beta} * f(y)\right|^{2} t^{\frac{\beta-1}{\beta}} d y d t\right)^{1 / 2}<\infty ;
\end{aligned}
$$

(ii) A tempered distribution $f$ on $\mathbb{R}^{n}$ belongs to $\overline{V B M O^{-(2 \beta-1)}}\left(\mathbb{R}^{n}\right)$ provided

$$
\lim _{T \longrightarrow 0}\|f\|_{B M O_{T}^{-(2 \beta-1)}\left(\mathbb{R}^{n}\right)}=0 ;
$$

(iii) A function $g$ on $\mathbb{R}_{+}^{1+n}$ belongs to the space $X_{T}^{\beta}\left(\mathbb{R}^{n}\right)$ provided

$$
\begin{aligned}
\|g\|_{X_{T}^{\beta}\left(\mathbb{R}^{n}\right)} & =\sup _{t \in(0, T)} t^{1-\frac{1}{2 \beta}}\|g(t, \cdot)\|_{L^{\infty}\left(\mathbb{R}^{n}\right)} \\
& +\sup _{x \in \mathbb{R}^{n}, r^{2 \beta} \in(0, T)}\left(r^{-n} \int_{0}^{r^{2 \beta}} \int_{|y-x|<r}|g(t, y)|^{2} t^{\frac{\beta-1}{\beta}} d y d t\right)^{1 / 2}<\infty .
\end{aligned}
$$

Using the fact when $\alpha=1-\beta, Q_{\alpha ; \infty}^{\beta,-1}\left(\mathbb{R}^{n}\right)=B M O^{-\zeta}\left(\mathbb{R}^{n}\right)$ for $\zeta=2 \beta-1$ and the boundedness of bilinear operator

$$
B(u, v)=\int_{0}^{t} e^{-(t-s)(-\triangle)^{\beta}} P \nabla \cdot(u \otimes v)(s) d s
$$

on the spaces $X_{T}^{\beta}\left(\mathbb{R}^{n}\right)$ for $T \in(0, \infty]$, see Li and Zhai's [14, Theorem 4.13], we can get the following result.

Theorem 1.8. Let $n \geq 2,1 / 2<\beta<1$. Then

(i) The fractional Navier-Stokes system (1.1) has a unique small global mild solution in $\left(X_{\infty}^{\beta}\left(\mathbb{R}^{n}\right)\right)^{n}$ for all initial data a with $\nabla \cdot a=0$ and $\|a\|_{\left(B M O_{\infty}^{-(2 \beta-1)}\left(\mathbb{R}^{n}\right)\right)^{n}}$ being small. 
(ii) For any $T \in(0, \infty)$, there is an $\varepsilon>0$ such that the fractional Navier-Stokes system (1.1) has a unique small mild solution in $\left(X_{T}^{\beta}\left(\mathbb{R}^{n}\right)\right)^{n}$ on $(0, T) \times \mathbb{R}^{n}$ when the initial data a satisfies $\nabla \cdot a=0$ and $\|a\|_{\left(B M O_{T}^{-(2 \beta-1)}\left(\mathbb{R}^{n}\right)\right)^{n}} \leq \varepsilon$. In particular for all $a \in\left(\overline{V B M O^{-(2 \beta-1)}}\left(\mathbb{R}^{n}\right)\right)^{n}$ with $\nabla \cdot a=0$ there exists a unique small local mild solution in $\left(X_{T}^{\beta}\left(\mathbb{R}^{n}\right)\right)^{n}$ on $(0, T) \times \mathbb{R}^{n}$.

Proposition 1.8 is an generalization of Koch and Tataru [13, Theorem 2-3] since

$$
B M O^{-(2 \beta-1)}\left(\mathbb{R}^{n}\right)=(-\triangle)^{-\frac{2 \beta-1}{2}} B M O\left(\mathbb{R}^{n}\right) .
$$

Similar to Proposition 1.8, we can consider the well-posedness for dissipative quasigeostrophic equations in $B M O^{-(2 \beta-1)}\left(\mathbb{R}^{2}\right)$.

As an application, we prove the existence of solutions to the fractional magnetohydrodynamics equations

$$
\begin{cases}\partial_{t} u+(-\triangle)^{\beta} u+u \cdot \nabla u+\nabla p-b \cdot \nabla b=0, & \text { in } \mathbb{R}_{+}^{1+n} \\ \partial_{t} b+(-\triangle)^{\beta} b+b \cdot \nabla b-b \cdot \nabla u=0, & \text { in } \mathbb{R}_{+}^{1+n} \\ \nabla \cdot u=\nabla \cdot b=0, & \text { in } \mathbb{R}_{+}^{1+n} \\ \left.u\right|_{t=0}=u_{0},\left.b\right|_{t=0}=b_{0}, & \text { in } \mathbb{R}^{n}\end{cases}
$$

We refer the readers to $\mathrm{Wu}[\mathbf{1 9}]$ and $[\mathbf{2 2}]$, Cao and $\mathrm{Wu}[\mathbf{4}]$, Zhou $[\mathbf{2 6}]$ and the references therein for more information about this system.

Propisition 1.9. Let $n \geq 2, \beta \in(1 / 2,1), \max \{2 \beta-1,2-2 \beta\}<w<2 \beta$, $1+n / p+w<4 \beta,\left(u_{0}, b_{0}\right) \in G_{n}^{-(2 \beta-1)}\left(\mathbb{R}^{n}\right), \nabla \cdot u_{0}=0$ and $\nabla \cdot b_{0}=0$. If $\left\|u_{0}\right\|_{G_{n}^{-(2 \beta-1)}\left(\mathbb{R}^{n}\right)}+\left\|b_{0}\right\|_{G_{n}^{-(2 \beta-1)}\left(\mathbb{R}^{n}\right)}$ is small enough, then there is a unique mild solution $(u, b)$ to (1.7) satisfying

$$
\begin{gathered}
\|u(t)\|_{G_{n}^{-(2 \beta-1)}\left(\mathbb{R}^{n}\right)}+t^{\frac{1}{2 \beta}}\|u(t)\|_{L^{\infty}\left(\mathbb{R}^{n}\right)}+t^{\frac{w}{2 \beta}}\|u(t)\|_{\dot{B}_{\infty, \infty}^{w-(2 \beta-1)}\left(\mathbb{R}^{n}\right)} \lesssim\|a\|_{G_{n}^{-(2 \beta-1)}\left(\mathbb{R}^{n}\right)}, \\
\|b(t)\|_{G_{n}^{-(2 \beta-1)}\left(\mathbb{R}^{n}\right)}+t^{\frac{1}{2 \beta}}\|b(t)\|_{L^{\infty}\left(\mathbb{R}^{n}\right)}+t^{\frac{w}{2 \beta}}\|b(t)\|_{\dot{B}_{\infty, \infty}^{w-(2 \beta-1)}\left(\mathbb{R}^{n}\right)} \lesssim\|a\|_{G_{n}^{-(2 \beta-1)}\left(\mathbb{R}^{n}\right)} .
\end{gathered}
$$

Using the boundedness of operator $B(u, v)$ on spaces $X_{T}^{\beta}\left(\mathbb{R}^{n}\right)$ for $T \in(0, \infty]$ and the contraction mapping principle, we get the well-posedness for system (1.7) with initial data in $\left(B M O_{T}^{-(2 \beta-1)}\left(\mathbb{R}^{n}\right)\right.$ even in more general classes $Q_{\alpha ; T}^{\beta,-1}\left(\mathbb{R}^{n}\right)$ for $T \in(0, \infty]$. Here, we only state the result about $\left(B M O_{T}^{-(2 \beta-1)}\left(\mathbb{R}^{n}\right)\right.$.

Propisition 1.10. Let $n \geq 2,1 / 2<\beta<1$. Then

(i) The system (1.7) has a unique small global mild solution $(u, b)$ in $\left(X_{\infty}^{\beta}\left(\mathbb{R}^{n}\right)\right)^{n} \times$ $\left(X_{\infty}^{\beta}\left(\mathbb{R}^{n}\right)\right)^{n}$ for all initial data $\left(u_{0}, b_{0}\right)$ with

$$
\left\|u_{0}\right\|_{\left(B M O_{\infty}^{-(2 \beta-1)}\left(\mathbb{R}^{n}\right)\right)^{n}} \text { and }\left\|b_{0}\right\|_{\left(B M O_{\infty}^{-(2 \beta-1)}\left(\mathbb{R}^{n}\right)\right)^{n}}
$$

small enough and

$$
\nabla \cdot u_{0}=\nabla \cdot b_{0}=0 .
$$

(ii) For any $T \in(0, \infty)$, there is an $\varepsilon>0$ such that the system (1.7) has a unique small mild solution $(u, b)$ in $\left(X_{T}^{\beta}\left(\mathbb{R}^{n}\right)\right)^{n} \times\left(X_{T}^{\beta}\left(\mathbb{R}^{n}\right)\right)^{n}$ when the initial data $\left(u_{0}, b_{0}\right)$ satisfies

$\nabla \cdot u_{0}=\nabla \cdot b_{0}=0, \quad\left\|u_{0}\right\|_{\left(B M O_{T}^{-(2 \beta-1)}\left(\mathbb{R}^{n}\right)\right)^{n}} \leq \varepsilon \quad$ and $\quad\left\|b_{0}\right\|_{\left(B M O_{T}^{-(2 \beta-1)}\left(\mathbb{R}^{n}\right)\right)^{n}} \leq \varepsilon$. In particular for all $u_{0} \in\left(\overline{V B M O^{-(2 \beta-1)}}\left(\mathbb{R}^{n}\right)\right)^{n}$ and $b_{0} \in\left(\overline{V B M O^{-(2 \beta-1)}}\left(\mathbb{R}^{n}\right)\right)^{n}$ with

$$
\nabla \cdot u_{0}=\nabla \cdot b_{0}=0
$$


there exists a unique small local mild solution $(u, b)$ in $\left(X_{T}^{\beta}\left(\mathbb{R}^{n}\right)\right)^{n} \times\left(X_{T}^{\beta}\left(\mathbb{R}^{n}\right)\right)^{n}$.

The rest of this paper is organized as follows. In Section 2, we give the definition and some basic properties of Besov spaces. In Section 3, we prove our main results. First we prove Proposition 1.2 - a prior estimates for fractional Navier-Stokes equations. Then, we verify Theorem 1.3 by applying Proposition 1.2 and demonstrate Proposition 1.4 by using the contraction mapping principle and Proposition 1.2. Meanwhile, Theorems 1.5 and 1.6 are shown by applying Littlewood-Paley theory. Finally, we show Proposition 1.9 by the contraction mapping principle.

\section{Preliminary lemmas}

In this section, we provide the definition and several properties of the homogeneous Besov spaces.

We recall the definition of homogeneous Besov spaces. For details, see Berg and Lofstrom [1] and Triebel [18]. We start with the fourier transform. The Fourier transform $\hat{f}$ of $f \in \mathcal{S}$ is defined as

$$
\widehat{f}(\xi)=(2 \pi)^{-n / 2} \int_{\mathbb{R}^{n}} f(x) e^{-x \cdot \xi} d x
$$

Here $\mathcal{S}\left(\mathbb{R}^{n}\right)$ denotes the Schwartz class of rapidly decreasing smooth functions and $\mathcal{S}^{\prime}\left(\mathbb{R}^{n}\right)$ is the space of tempered distributions. The fractional power of the Laplacian can be defined by the Fourier transform. For $\theta \in \mathbb{R}$,

$$
\left(-\widehat{\triangle)^{\theta / 2}} f(\xi)=|\xi|^{\theta} \widehat{f}(\xi)\right.
$$

We will use $f^{\vee}$ to denote the inverse Fourier transform of $f$. Then we introduce the Littlewood-Paley decomposition by means of $\left\{\varphi_{j}\right\}_{j=-\infty}^{\infty}$. Take a function $\phi \in C_{0}^{\infty}$ with

$$
\operatorname{supp}(\phi)=\left\{\xi \in \mathbb{R}^{n}: 1 / 2<|\xi| \leq 2\right\}
$$

such that $\sum_{j=-\infty}^{\infty} \phi\left(2^{-j} \xi\right)=1$ for all $\xi \neq 0$. Then we define functions $\varphi_{j}(j=$ $0, \pm 1, \pm 2, \cdots)$ as

$$
\widehat{\varphi_{j}}(\xi)=\phi\left(2^{-j} \xi\right)
$$

Let $\triangle_{j} f=\varphi_{j} * f$, for $j=0, \pm 1, \pm 2, \pm 3, \cdots$. Then, for $s \in \mathbb{R}$ and $1 \leq p, q \leq \infty$, we define

$$
\begin{gathered}
\|f\|_{\dot{B}_{p, q}^{s}\left(\mathbb{R}^{n}\right)}=\left(\sum_{j=-\infty}^{\infty}\left(2^{s j}\left\|\triangle_{j} f\right\|_{L^{p}\left(\mathbb{R}^{n}\right)}\right)^{q}\right)^{1 / q}, \quad 1 \leq q<\infty \\
\|f\|_{\dot{B}_{p, \infty}^{s}\left(\mathbb{R}^{n}\right)}=\sup _{-\infty<j<\infty}\left(2^{s j}\left\|\triangle_{j} f\right\|_{L^{p}\left(\mathbb{R}^{n}\right)}\right), \quad q=\infty,
\end{gathered}
$$

where $L^{p}\left(\mathbb{R}^{n}\right)$ means the usual Lebesgue space on $\mathbb{R}^{n}$ with the norm $\|\cdot\|_{L^{p}\left(\mathbb{R}^{n}\right)}$. The homogeneous Bosev space $\dot{B}_{p, q}^{s}\left(\mathbb{R}^{n}\right)$ is defined by

$$
\dot{B}_{p, q}^{s}\left(\mathbb{R}^{n}\right)=\left\{f \in \mathcal{S}^{\prime}:\|f\|_{\dot{B}_{p, q}^{s}\left(\mathbb{R}^{n}\right)}<\infty\right\} .
$$

We will use the following properties about homogeneous Besov space.

LEMMA 2.1. The following properties hold:

(i) If $1 \leq q_{1} \leq q_{2} \leq \infty, 1 \leq p \leq \infty$ and $s \in \mathbb{R}$, then $\dot{B}_{p, q_{1}}^{s}\left(\mathbb{R}^{n}\right) \hookrightarrow \dot{B}_{p, q_{2}}^{s}\left(\mathbb{R}^{n}\right)$.

(ii) If $1 \leq p_{1} \leq p_{2} \leq \infty, 1 \leq q \leq \infty,-\infty<s_{1} \leq s_{2}<\infty$ and $s_{2}-\frac{n}{p_{2}}=s_{1}-\frac{n}{p_{1}}$, then

$$
\dot{B}_{p_{2}, q}^{s_{2}}\left(\mathbb{R}^{n}\right) \hookrightarrow \dot{B}_{p_{1}, q}^{s_{1}}\left(\mathbb{R}^{n}\right)
$$


(iii) If $\beta, s \in \mathbb{R}, 1 \leq p, q \leq \infty$, then the operator $(-\triangle)^{\beta / 2}$ is an isomorphism from $\dot{B}_{p, q}^{s}\left(\mathbb{R}^{n}\right)$ to $\dot{B}_{p, q}^{s-\beta}\left(\mathbb{R}^{n}\right)$.

LEMMA 2.2. Let $0<\theta<1,1 \leq p, q \leq \infty,-\infty<s_{1}<s_{2}<\infty$ and $s=$ $(1-\theta) s_{1}+\theta s_{2}$. Then

$$
\left(\dot{B}_{p, \infty}^{s_{1}}\left(\mathbb{R}^{n}\right), \dot{B}_{p, \infty}^{s_{2}}\left(\mathbb{R}^{n}\right)\right)_{\theta, q}=\dot{B}_{p, q}^{s}\left(\mathbb{R}^{n}\right)
$$

for $s=s_{1}(1-\theta)+s_{2} \theta$, where $(\cdot, \cdot)_{\theta, q}$ means the real interpolation functor, see Berg and Lofstrom [1].

We will use the $L^{p}-L^{q}$-type estimates for $e^{-t(-\triangle)^{\theta}}$ in homogeneous Besov spaces. For $\theta=1$, the $L^{p}-L^{p}$-estimates for $e^{t \triangle}$ in Besov spaces were studied by Kozono, Ogawa and Taniuchi in [11]. Zhai in [25] proved the general case of $\theta>0$.

LEMMA 2.3. Let $\theta>0$ and $\zeta \geq 0$. If $s_{1} \leq s_{2}, 1 \leq p_{1} \leq p_{2} \leq \infty$ and $1 \leq q \leq \infty$, then

$$
\left\|e^{-t(-\triangle)^{\theta}} f\right\|_{\dot{B}_{p_{2}, q}^{s_{2}}\left(\mathbb{R}^{n}\right)} \lesssim t^{-\frac{s_{2}-s_{1}}{2 \theta}-\frac{n}{2 \theta}\left(\frac{1}{p_{1}}-\frac{1}{p_{2}}\right)}\|f\|_{\dot{B}_{p_{1}, q}^{s_{1}}\left(\mathbb{R}^{n}\right)} .
$$

The following equivalent characterization of homogeneous Besov spaces will be useful.

LEMMA 2.4. ([18]) Let $0<s<1$ and $1 \leq p \leq \infty$, then in $\dot{B}_{p, \infty}^{s}\left(\mathbb{R}^{n}\right)$, we have

$$
\|f\|_{\dot{B}_{p, \infty}^{s}\left(\mathbb{R}^{n}\right)} \equiv \sup _{y \neq 0} \frac{\|f(\cdot+y)-u(\cdot)\|_{L^{p}\left(\mathbb{R}^{n}\right)}}{|y|^{s}} .
$$

We need a variant of Mikhlin theorem on Fourier multipliers.

LEMMA 2.5. ([18]) Let $-\infty<s<\infty$ and $\phi(x)$ be a complex-valued infinitely differentiable function on $\mathbb{R}^{n} \backslash\{0\}$ so that

$$
\sup _{j \leq k} \sup _{x \in \mathbb{R}^{n}}|x|^{j}\left|\nabla^{j} \phi(x)\right|<\infty
$$

for a sufficiently large positive integer $k$. Then

$$
\left\|(\phi \widehat{u})^{\vee}\right\|_{\dot{B}_{p, q}^{s}\left(\mathbb{R}^{n}\right)} \lesssim\|u\|_{\dot{B}_{p, q}^{s}\left(\mathbb{R}^{n}\right)}
$$

for $u \in \dot{B}_{p, q}^{s}\left(\mathbb{R}^{n}\right)$ with $1 \leq p, q \leq \infty$.

We need a useful lemma, see Grafakos [10], Frazier, Jawerth and Weiss [8].

LEMMA 2.6. Let $f \in \mathcal{S}^{\prime}\left(\mathbb{R}^{n}\right)$. Then the following statements are equivalent:

(i) $f \in B M O\left(\mathbb{R}^{n}\right)$;

(ii) for all $\phi \in \mathcal{S}^{\prime}\left(\mathbb{R}^{n}\right)$ satisfying:

$$
\int_{\mathbb{R}^{n}} \phi(x) d x=0, \quad \sup _{\xi \in \mathbb{R}^{n}} \int_{0}^{\infty}|\widehat{\phi}(t \xi)|^{2} \frac{d t d \xi}{t}<\infty
$$

and $|\phi(x)| \lesssim \frac{1}{(1+|x|)^{n+c}}$ for some $c$, then the measure $d \mu(t, x)=\left|\phi_{t} * b(x)\right|^{2} \frac{d t d x}{t}$ is a Carleson measure on $\mathbb{R}_{+}^{1+n}$.

Lemma 2.7. Let $2 \beta-1<w<2 \beta, 2 \leq n \leq p \leq \infty, 1 \leq q \leq \infty$, then one has

$$
\begin{aligned}
& t^{\frac{2 \beta-1}{2 \beta}}\|u(t)\|_{L^{\infty}\left(\mathbb{R}^{n}\right)}+t^{\frac{w-(2 \beta-1)}{2 \beta}}\|u(t)\|_{\dot{B}_{p, \infty}^{w-2 \beta+\frac{n}{p}}}\left(\mathbb{R}^{n}\right) \\
& \lesssim\|u(t)\|_{\dot{B}_{p, \infty}^{-(2 \beta-1)+\frac{n}{p}}\left(\mathbb{R}^{n}\right)}+t^{\frac{w}{2 \beta}}\|u(t)\|_{\dot{B}_{p, \infty}^{w-(2 \beta-1)+\frac{n}{p}}\left(\mathbb{R}^{n}\right)} .
\end{aligned}
$$


Proof. It follows from Lemmas 2.1-2.2 and [18, Proposition 2.5.7] that

$$
\dot{B}_{p, \infty}^{w-2 \beta}\left(\mathbb{R}^{n}\right)=\left(\dot{B}_{p, \infty}^{-(2 \beta-1)}\left(\mathbb{R}^{n}\right), \dot{B}_{p, \infty}^{w-(2 \beta-1)}\left(\mathbb{R}^{n}\right)\right)_{\frac{w-1}{w}, \infty}
$$

and

$$
\dot{B}_{\infty, \infty}^{0}\left(\mathbb{R}^{n}\right) \supset L^{\infty}\left(\mathbb{R}^{n}\right) \supset \dot{B}_{\infty, 1}^{0}\left(\mathbb{R}^{n}\right)=\left(\dot{B}_{\infty, \infty}^{-(2 \beta-1)}\left(\mathbb{R}^{n}\right), \dot{B}_{\infty, \infty}^{w-(2 \beta-1)}\left(\mathbb{R}^{n}\right)\right)_{\frac{2 \beta-1}{w}, 1}
$$

which contains

$$
\left(\dot{B}_{p, \infty}^{-(2 \beta-1)+\frac{n}{p}}\left(\mathbb{R}^{n}\right), \dot{B}_{p, \infty}^{w-(2 \beta-1)+\frac{n}{p}}\left(\mathbb{R}^{n}\right)\right)_{\frac{2 \beta-1}{w}, 1}
$$

Hence, one has

$$
\begin{aligned}
& \left\|u\left(t, t^{\frac{1}{2 \beta}} \cdot\right)\right\|_{L^{\infty}\left(\mathbb{R}^{n}\right)}+\left\|u\left(t, t^{\frac{1}{2 \beta}} \cdot\right)\right\|_{\dot{B}_{p, \infty}^{w-2 \beta+\frac{n}{p}}\left(\mathbb{R}^{n}\right)} \\
& \lesssim\left\|u\left(t, t^{\frac{1}{2 \beta}} \cdot\right)\right\|_{\dot{B}_{p, \infty}^{-(2 \beta-1)+\frac{n}{p}}\left(\mathbb{R}^{n}\right)}+\left\|u\left(t, t^{\frac{1}{2 \beta}} \cdot\right)\right\|_{\dot{B}_{p, \infty}^{w-(2 \beta-1)+\frac{n}{p}}\left(\mathbb{R}^{n}\right)} .
\end{aligned}
$$

By changing variables, we get

$$
\begin{aligned}
& t^{\frac{2 \beta-1}{2 \beta}}\|u(t)\|_{L^{\infty}\left(\mathbb{R}^{n}\right)}+t^{\frac{w-(2 \beta-1)}{2 \beta}}\|u(t)\|_{\dot{B}_{p, \infty}^{w-2 \beta+\frac{n}{p}}\left(\mathbb{R}^{n}\right)} \\
& \lesssim\|u(t)\|_{\dot{B}_{p, \infty}^{-(2 \beta-1)+\frac{n}{p}}\left(\mathbb{R}^{n}\right)}+t^{\frac{w}{2 \beta}}\|u(t)\|_{\dot{B}_{p, \infty}^{w-(2 \beta-1)+\frac{n}{p}}\left(\mathbb{R}^{n}\right)}
\end{aligned}
$$

LEMma 2.8. For $\beta \in(1 / 2,1), u, v \in\left(L^{\infty}\left(\mathbb{R}^{n}\right)\right)^{n} \cap\left(G_{n}^{-(2 \beta-1)}\left(\mathbb{R}^{n}\right)\right)^{n}$, one has

$$
\left\|e^{-t(-\triangle)^{\beta}} P \nabla \cdot(u \otimes v)\right\|_{G_{n}^{-(2 \beta-1)}\left(\mathbb{R}^{n}\right)} \lesssim t^{\frac{1-2 \beta}{2 \beta}}\|u\|_{L^{\infty}\left(\mathbb{R}^{n}\right)}\|v\|_{G_{n}^{-(2 \beta-1)}\left(\mathbb{R}^{n}\right)}
$$

Proof. It is easy to see that for $\beta \in(1 / 2,1)$,

$$
\left\|\partial_{x_{i}} \partial_{x_{j}} \triangle^{-1} \partial_{x_{k}} K_{t}^{\beta}(x)\right\|_{L^{1}\left(\mathbb{R}^{n}\right)} \lesssim t^{-\frac{1}{2 \beta}}(i, j, k=1,2, \cdots, n)
$$

Since the operation with respect to the convolution is commutative, by letting

$$
K_{i, j, k, t}=\left(\delta_{i j}-\partial_{x_{i}} \partial_{x_{j}} \Delta^{-1}\right) \partial_{x_{k}} K_{t}^{\beta}(x),
$$


one has, for $s>0$,

$$
\begin{aligned}
& \left\|e^{-s(-\triangle)^{\beta}}\left|e^{-t(-\triangle)^{\beta}} P \nabla \cdot(u \otimes v)\right|\right\|_{L^{\infty}\left(\mathbb{R}^{n}\right)} \\
\leq & \sum_{i=1}^{n} \sum_{j=1}^{n} \sum_{k=1}^{n}\left\|K_{s}^{\beta} *\left|K_{i, j, k, t} *\left(u_{k} v_{j}\right)\right|\right\|_{L^{\infty}\left(\mathbb{R}^{n}\right)} \\
\leq & \sum_{i=1}^{n} \sum_{j=1}^{n} \sum_{k=1}^{n}\left\|K_{s}^{\beta} *\left|K_{i, j, k, t}\right| *\left|u_{k} v_{j}\right|\right\|_{L^{\infty}\left(\mathbb{R}^{n}\right)} \\
\leq & \sum_{i=1}^{n} \sum_{j=1}^{n} \sum_{k=1}^{n}\left\|\left|K_{i, j, k, t} * K_{s}^{\beta} *\right| u_{k} v_{j} \mid\right\|_{L^{\infty}\left(\mathbb{R}^{n}\right)} \\
\leq & \sum_{i=1}^{n} \sum_{j=1}^{n} \sum_{k=1}^{n}\left\|K_{i, j, k, t}\right\|_{L^{1}\left(\mathbb{R}^{n}\right)}\left\|K_{s}^{\beta} *\left|u_{k} v_{j}\right|\right\|_{L^{\infty}\left(\mathbb{R}^{n}\right)} \\
\leq & \left(\left\|\nabla K_{t}^{\beta}\right\|_{L^{1}\left(\mathbb{R}^{n}\right)}+\sum_{i=1}^{n} \sum_{j=1}^{n} \sum_{k=1}^{n}\left\|\partial_{x_{i}} \partial_{x_{j}} \triangle^{-1} \partial_{x_{k}} K_{t}^{\beta}\right\|_{L^{1}\left(\mathbb{R}^{n}\right)}\right) \\
& \left\|e^{-s(-\triangle)^{\beta}}\left|u_{k} v_{j}\right|\right\|_{L^{\infty}\left(\mathbb{R}^{n}\right)} \\
\lesssim & t^{-\frac{1}{2 \beta}}\|u\|_{L^{\infty}\left(\mathbb{R}^{n}\right)} \| e^{-s(-\Delta)^{\beta}|v| \|_{L^{\infty}\left(\mathbb{R}^{n}\right)} .}
\end{aligned}
$$

So,

$$
\begin{aligned}
& \sup _{s>0} s^{\frac{2 \beta-1}{2 \beta}}\left\|e^{-s(-\triangle)}\left|e^{-t(-\triangle)^{\beta}} P \nabla \cdot(u \otimes v)\right|\right\|_{L^{\infty}\left(\mathbb{R}^{n}\right)} \\
& \lesssim t^{-\frac{1}{2 \beta}}\|u\|_{L^{\infty}\left(\mathbb{R}^{n}\right)} \sup _{s>0} s^{\frac{2 \beta-1}{2 \beta}}\left\|e^{-s(-\triangle)^{\beta}}|v|\right\|_{L^{\infty}\left(\mathbb{R}^{n}\right)} .
\end{aligned}
$$

\section{Proof of main results}

3.1. Proof of Proposition 1.2 It follows from Lemma 2.5 that

$$
\|P v\|_{\dot{B}_{p, q}^{s}\left(\mathbb{R}^{n}\right)}+\left\|\nabla(-\triangle)^{-1 / 2} v\right\|_{\dot{B}_{p, q}^{s}\left(\mathbb{R}^{n}\right)} \lesssim\|v\|_{\dot{B}_{p, q}^{s}\left(\mathbb{R}^{n}\right)} .
$$

On the other hand, it is easy to see that for $k \geq 0$,

$$
\left\|\nabla^{k} e^{-t(-\triangle)^{\beta}} v\right\|_{L^{p}\left(\mathbb{R}^{n}\right)} \lesssim t^{-\frac{k}{2 \beta}}\|v\|_{L^{p}\left(\mathbb{R}^{n}\right)} .
$$

Then (iii) of Lemma 2.1 tells us 


$$
\begin{aligned}
& \left\|u(t)-e^{-t(-\triangle)^{\beta}} a\right\|_{\dot{B}_{p, \infty}^{w-(2 \beta-1)+\frac{n}{p}}\left(\mathbb{R}^{n}\right)} \\
& \lesssim \sup _{\tau>0} \frac{2 \beta-\left[w-(2 \beta-1)+\frac{n}{p}\right]}{2 \beta} \\
& \left\|\triangle^{\beta+1} \int_{0}^{t} e^{-(t-s+\tau)(-\triangle)^{\beta}} \triangle^{-1} P \nabla \cdot f(s) d s\right\|_{L^{p}\left(\mathbb{R}^{n}\right)} \\
& \lesssim \sup _{\tau>0} \frac{2 \beta-\left[w-(2 \beta-1)+\frac{n}{p}\right]}{2 \beta} \int_{0}^{t}(t+\tau-s)^{-\frac{2 \beta}{2 \beta}} \\
& \left\|\triangle e^{-\frac{(t+\tau-s)}{2}(-\triangle)^{\beta}} \triangle^{-1} P \nabla \cdot f(s)\right\|_{L^{p}\left(\mathbb{R}^{n}\right)} d s \\
& \lesssim \sup _{\tau>0} \frac{2 \beta-\left[w-(2 \beta-1)+\frac{n}{p}\right]}{2 \beta} \int_{0}^{t}(t+\tau-s) \frac{-4 \beta+\left[w-(2 \beta-1)+\frac{n}{p}\right]}{2 \beta} \\
& \left\|\triangle^{-1} P \nabla \cdot f(s)\right\|_{\dot{B}_{p, \infty}^{w-(2 \beta-1)+\frac{n}{p}}\left(\mathbb{R}^{n}\right)} d s \\
& \lesssim \sup _{\tau>0} \frac{2 \beta-\left[w-(2 \beta-1)+\frac{n}{p}\right]}{2 \beta} \int_{0}^{t}(t+\tau-s)^{\frac{-4 \beta+\left[w-(2 \beta-1)+\frac{n}{p}\right]}{2 \beta}} \\
& \left\|P(-\triangle)^{-1 / 2} \nabla \cdot f(s)\right\|_{\dot{B}_{p, \infty}^{w-2 \beta+\frac{n}{p}}\left(\mathbb{R}^{n}\right)} d s .
\end{aligned}
$$

This implies

$$
\begin{aligned}
& \left\|u(t)-e^{-t(-\triangle)^{\beta}} a\right\|_{\dot{B}_{p, \infty}^{w-(2 \beta-1)+\frac{n}{p}}}\left(\mathbb{R}^{n}\right) \\
& \lesssim \sup _{\tau>0} \frac{2 \beta-\left[w-(2 \beta-1)+\frac{n}{p}\right]}{2 \beta} \int_{0}^{t}(t+\tau-s)^{\frac{-4 \beta+\left[w-(2 \beta-1)+\frac{n}{p}\right]}{2 \beta}}\|f(s)\|_{\dot{B}_{p, \infty}^{w-2 \beta+\frac{n}{p}}\left(\mathbb{R}^{n}\right)} d s \\
& \lesssim \sup _{\tau>0} \frac{2 \beta-\left[w-(2 \beta-1)+\frac{n}{p}\right]}{2 \beta}\left(\int_{0}^{t / 2}+\int_{t / 2}^{t}\right)(t+\tau-s)^{\frac{-4 \beta+\left[w-(2 \beta-1)+\frac{n}{p}\right]}{2 \beta}} s^{-\frac{w}{2 \beta}} d s \\
& \times \sup _{0<s<t} s^{\frac{w}{2 \beta}}\|f(s)\|_{\dot{B}_{p, \infty}^{w-2 \beta+\frac{n}{p}}}\left(\mathbb{R}^{n}\right) \\
& \lesssim \sup _{\tau>0} \frac{2 \beta-\left[w-(2 \beta-1)+\frac{n}{p}\right]}{2 \beta} t^{-\frac{w}{2 \beta}} \int_{0}^{t}(t+\tau-s)^{\frac{-4 \beta+\left[w-(2 \beta-1)+\frac{n}{p}\right]}{2 \beta}} d s \\
& \times \sup _{0<s<t} s^{-\frac{w}{2 \beta}}\|f(s)\|_{\dot{B}_{p, \infty}^{w-2 \beta+\frac{n}{p}}}\left(\mathbb{R}^{n}\right) \\
& +\sup _{\tau>0} \frac{2 \beta-\left[w-(2 \beta-1)+\frac{n}{p}\right]}{2 \beta}(t+\tau) \frac{-4 \beta+\left[w-(2 \beta-1)+\frac{n}{p}\right]}{2 \beta} \int_{0}^{t} s^{-\frac{w}{2 \beta}} d s \\
& \times \sup _{0<s<t} s^{\frac{w}{2 \beta}}\|f(s)\|_{\dot{B}_{p, \infty}^{w-2 \beta+\frac{n}{p}}\left(\mathbb{R}^{n}\right)} \\
& \lesssim \sup _{\tau>0} \tau^{2 \beta-\left[w-(2 \beta-1)+\frac{n}{p}\right]} t^{-\frac{w}{2 \beta}} \int_{0}^{t}(t+\tau-s)^{\frac{-4 \beta+\left[w-(2 \beta-1)+\frac{n}{p}\right]}{2 \beta}} d s \\
& \times \sup _{0<s<t} s^{-\frac{w}{2 \beta}}\|f(s)\|_{\dot{B}_{p, \infty}^{w-2 \beta+\frac{n}{p}}}\left(\mathbb{R}^{n}\right)
\end{aligned}
$$




$$
\begin{aligned}
&+\sup _{\tau>0}(t+\tau) \frac{2 \beta-\left[w-(2 \beta-1)+\frac{n}{p}\right]}{2 \beta}+\frac{-4 \beta+\left[w-(2 \beta-1)+\frac{n}{p}\right]}{2 \beta} \int_{0}^{t} s^{-\frac{w}{2 \beta}} d s \\
& \times \sup _{0<s<t} s^{\frac{w}{2 \beta}}\|f(s)\|_{\dot{B}_{p, \infty}^{w-2 \beta+\frac{n}{p}}\left(\mathbb{R}^{n}\right)} \\
& \lesssim \sup _{\tau>0} \frac{2 \beta-\left[w-(2 \beta-1)+\frac{n}{p}\right]}{2 \beta} t^{-\frac{w}{2 \beta}} \int_{0}^{t}(t+\tau-s) \frac{-4 \beta+\left[w-(2 \beta-1)+\frac{n}{p}\right]}{2 \beta} d s \\
& \quad \times \sup _{0<s<t} s^{-\frac{w}{2 \beta}}\|f(s)\|_{\dot{B}_{p, \infty}^{w-2 \beta+\frac{n}{p}}}\left(\mathbb{R}^{n}\right) \\
& \quad+\sup _{\tau>0}(t+\tau)^{-1} t^{1-\frac{w}{2 \beta}} \times \sup _{0<s<t} s^{\frac{w}{2 \beta}}\|f(s)\|_{\dot{B}_{p, \infty}^{w-2 \beta+\frac{n}{p}}\left(\mathbb{R}^{n}\right)} \\
& \lesssim \quad t^{-\frac{w}{2 \beta}} \sup _{0<s<t} s^{\frac{w}{2 \beta}}\|f(s)\|_{\dot{B}_{p, \infty}^{w-2 \beta+\frac{n}{p}}}\left(\mathbb{R}^{n}\right)
\end{aligned}
$$

since $1+n / p+w<4 \beta$. Thus, by (iii) of Lemma 2.1 and Lemma 2.3, one has, for $2-2 \beta<w<2 \beta<2$,

$$
\begin{aligned}
& \left\|u(t)-e^{-t(-\triangle)^{\beta}} a\right\|_{\dot{B}_{p, q}^{-(2 \beta-1)+\frac{n}{p}}\left(\mathbb{R}^{n}\right)} \\
\lesssim & \int_{0}^{t}\left\|e^{-(t-s)(-\triangle)^{\beta}} \nabla \cdot f(s)\right\|_{\dot{B}_{p, q}^{-(2 \beta-1)+\frac{n}{p}}\left(\mathbb{R}^{n}\right)} d s \\
\lesssim & \int_{0}^{t}(t-s)^{-\frac{2-w}{2 \beta}}\|f(s)\|_{\dot{B}_{p, q}^{w-2 \beta+\frac{n}{p}}\left(\mathbb{R}^{n}\right)} d s \\
\lesssim & \int_{0}^{t}(t-s)^{-\frac{2-w}{2 \beta}} s^{-\frac{w}{2 \beta}-\left(1-\frac{1}{\beta}\right)} \sup _{0<s, t} s^{\frac{w}{2 \beta}+\left(1-\frac{1}{\beta}\right)}\|f(s)\|_{\dot{B}_{n, \infty}^{w-2 \beta+\frac{n}{p}}\left(\mathbb{R}^{n}\right)} \\
\lesssim & \sup _{0<s, t} s^{\frac{w}{2 \beta}+1-\frac{1}{\beta}}\|f(s)\|_{\dot{B}_{n, \infty}^{w-2 \beta+\frac{n}{p}}}\left(\mathbb{R}^{n}\right)
\end{aligned}
$$

A combination of the previous estimates implies

$$
\begin{aligned}
& t^{\frac{w}{2 \beta}}\|u(t)\|_{\dot{B}_{p, \infty}^{w-(2 \beta-1)+\frac{n}{p}}\left(\mathbb{R}^{n}\right)} \\
& \lesssim t^{\frac{w}{2 \beta}}\left\|e^{-t(-\Delta)^{\beta}} a\right\|_{\dot{B}_{p, \infty}^{w-(2 \beta-1)+\frac{n}{p}}\left(\mathbb{R}^{n}\right)}+\sup _{0<s<t} s^{\frac{w}{2 \beta}}\|f(s)\|_{\dot{B}_{p, \infty}^{w-2 \beta+\frac{n}{p}}\left(\mathbb{R}^{n}\right)},
\end{aligned}
$$

and

$$
\begin{aligned}
& \|u(t)\|_{\dot{B}_{p, q}^{-(2 \beta-1)+\frac{n}{p}}\left(\mathbb{R}^{n}\right)} \\
& \lesssim\left\|e^{-t(-\triangle)^{\beta}} a\right\|_{\dot{B}_{p, q}^{-(2 \beta-1)+\frac{n}{p}}\left(\mathbb{R}^{n}\right)}+\sup _{0<s<t} s^{\frac{w}{2 \beta}+1-\frac{1}{\beta}}\|f(s)\|_{\dot{B}_{p, \infty}^{w-2 \beta+\frac{n}{p}}\left(\mathbb{R}^{n}\right)} .
\end{aligned}
$$

Thus we can get our estimates by applying Lemma 2.3 and inequality (3.1).

3.2. Proof of Theorem 1.3 Define

$$
X=\left\{u:[0, \infty) \longrightarrow G_{n}^{-(2 \beta-1)}\left(\mathbb{R}^{n}\right) \mid \nabla \cdot u=0,\|u\|_{X}<\infty\right\}
$$

with

$$
\|u\|_{X}=\sup _{t>0}\left(\|u(t)\|_{G_{n}^{-(2 \beta-1)}\left(\mathbb{R}^{n}\right)}+t^{\frac{w}{2 \beta}}\|u(t)\|_{\dot{B}_{\infty, \infty}^{w-(2 \beta-1)}\left(\mathbb{R}^{n}\right)}\right) .
$$

Set

$$
T(u)(t)=e^{-t(-\triangle)^{\beta}} a-\int_{0}^{t} e^{-(t-s)(-\triangle)^{\beta}} P \nabla \cdot(u(s) \otimes v(s)) d s .
$$


We want to show that $T$ is a contraction mapping from a ball of $X$ to itself. The case of $p=\infty$ in Lemma 2.7 implies that

$$
\begin{aligned}
& t^{\frac{w-2 \beta}{2 \beta}}\|v(t)\|_{\dot{B}_{\infty, \infty}^{w-(2 \beta-1)}\left(\mathbb{R}^{n}\right)}+t^{\frac{2 \beta-1}{2 \beta}}\|v(t)\|_{L^{\infty}\left(\mathbb{R}^{n}\right)} \\
& \lesssim\|v(t)\|_{\dot{B}_{\infty, \infty}^{-(2 \beta-1)}\left(\mathbb{R}^{n}\right)}+t^{\frac{w}{2 \beta}}\|v(t)\|_{\dot{B}_{\infty, \infty}^{w-(2 \beta-1)}\left(\mathbb{R}^{n}\right)} .
\end{aligned}
$$

Then, Proposition 1.2 and Lemma 2.4 imply

$$
\begin{aligned}
& t^{\frac{w}{2 \beta}}\|(T u)(t)\|_{\dot{B}_{\infty, \infty}^{w-(2 \beta-1)}\left(\mathbb{R}^{n}\right)} \\
\lesssim & \|a\|_{\dot{B}_{\infty, \infty}^{-(2 \beta-1)}\left(\mathbb{R}^{n}\right)}+\sup _{0<s<t} s^{\frac{w}{2 \beta}}\|u(s) \otimes u(s)\|_{\dot{B}_{\infty, \infty}^{w-2 \beta}\left(\mathbb{R}^{n}\right)} \\
\lesssim & \|a\|_{\dot{B}_{\infty, \infty}^{-(2 \beta-1)}\left(\mathbb{R}^{n}\right)}+\sup _{0<s<t} s^{\frac{2 \beta-1}{2 \beta}}\|u(s) \otimes v(s)\|_{\dot{B}_{\infty, \infty}^{-(2 \beta-1)}\left(\mathbb{R}^{n}\right)} \\
& +\sup _{0<s<t} s^{\frac{w+2 \beta-1}{2 \beta}}\|u(s) \otimes u(s)\|_{\dot{B}_{\infty, \infty}^{w-(2 \beta-1)}\left(\mathbb{R}^{n}\right)} \\
\lesssim & \|a\|_{\dot{B}_{\infty}^{-(2 \beta-1)}\left(\mathbb{R}^{n}\right)}+\sup _{0<s<t} s^{\frac{2 \beta-1}{2 \beta}}\|u(s) \otimes v(s)\|_{G_{n}^{-(2 \beta-1)}\left(\mathbb{R}^{n}\right)} \\
& +\sup _{0<s<t} s^{\frac{w+2 \beta-1}{2 \beta}}\|u(s) \otimes u(s)\|_{\dot{B}_{\infty, \infty}^{w-(2 \beta-1)}\left(\mathbb{R}^{n}\right)} \\
\lesssim & \|a\|_{\dot{B}_{\infty, \infty}^{-(2 \beta-1)}\left(\mathbb{R}^{n}\right)}+\sup _{0<s<t} s^{\frac{2 \beta-1}{2 \beta}}\|u(s)\|_{L^{\infty}\left(\mathbb{R}^{n}\right)}\|v(s)\|_{G_{n}^{-(2 \beta-1)}\left(\mathbb{R}^{n}\right)} \\
& +\sup _{0<s<t} s^{\frac{w+2 \beta-1}{2 \beta}}\|u(s)\|_{L^{\infty}\left(\mathbb{R}^{n}\right)}\|u(s)\|_{\dot{B}_{\infty, \infty}^{w-(2 \beta-1)}\left(\mathbb{R}^{n}\right)} \\
\lesssim & \|a\|_{G_{n}^{-(2 \beta-1)}\left(\mathbb{R}^{n}\right)}+\|u\|_{X}^{2} .
\end{aligned}
$$

On the other hand, Lemma 2.8 tells us

$$
\begin{aligned}
& \|(T u)(t)\|_{G_{n}^{w-(2 \beta-1)}\left(\mathbb{R}^{n}\right)} \\
\lesssim & \sup _{s>0} s^{\frac{2 \beta-1}{2 \beta}}\left\|e^{-t(-\triangle)^{\beta}} e^{-s(-\triangle)^{b e t a}}|a|\right\|_{L^{\infty}\left(\mathbb{R}^{n}\right)} \\
& +\int_{0}^{t}\left\|e^{-(t-s)(-\triangle)^{\beta}} P \nabla \cdot(u(s) \otimes u(s))\right\|_{G_{n}^{-(2 \beta-1)}\left(\mathbb{R}^{n}\right)} d s \\
\lesssim & \|a\|_{G_{n}^{-(2 \beta-1)}\left(\mathbb{R}^{n}\right)}+\int_{0}^{t}(t-s)^{\frac{2 \beta-1}{2 \beta}}\|u(s)\|_{G_{n}^{-(2 \beta-1)}\left(\mathbb{R}^{n}\right)}\|u(s)\|_{L^{\infty}\left(\mathbb{R}^{n}\right)} \\
\lesssim & \|a\|_{G_{n}^{-(2 \beta-1)}\left(\mathbb{R}^{n}\right)}+\|u\|_{X}^{2} .
\end{aligned}
$$

Hence, one has

$\|T u(t)\|_{X} \lesssim\|a\|_{G_{n}^{-(2 \beta-1)}\left(\mathbb{R}^{n}\right)}+\|u\|_{X}^{2}$ and $\|T u-T v\|_{X} \lesssim\left(\|u\|_{X}+\|v\|_{X}\right)\|u-v\|_{X}$.

Therefore, the contraction mapping principle implies that there exists a unique solution to equations (1.1) if $\|a\|_{G_{n}^{-(2 \beta-1)}\left(\mathbb{R}^{n}\right)}$ is small enough.

\subsection{Proof of Proposition 1.4 Define}

$$
K=\left\{f \in L^{\infty}\left((0, \infty) ; \dot{B}_{p, \infty}^{-(2 \beta-1)+\frac{n}{p}}\left(\mathbb{R}^{n}\right)\right): \nabla \cdot f=0,\|f\|_{K}<\infty\right\}
$$

with

$$
\|f\|_{K}=\sup _{t>0}\left(\|u(t)\|_{\dot{B}_{p, \infty}^{-(2 \beta-1)+\frac{n}{p}}\left(\mathbb{R}^{n}\right)}+t^{\frac{w}{2 \beta}}\|u(t)\|_{\dot{B}_{p, \infty}^{w-(2 \beta-1)+\frac{n}{p}}\left(\mathbb{R}^{n}\right)}\right) .
$$


Let

$$
T u(t)=e^{-t(-\triangle)^{\beta}} a-\int_{0}^{t} e^{-(t-s)(-\triangle)^{\beta}} P \nabla \cdot(u(s) \otimes u(s)) d s .
$$

We want to prove that $T$ is a contraction mapping from a ball of $K$ to itself. It follows from Lemmas 2.4 and 2.7 that

$$
\begin{aligned}
& \|T u(t)\|_{\dot{B}_{p, \infty}^{-(2 \beta-1)+\frac{n}{p}}\left(\mathbb{R}^{n}\right)}+t^{\frac{w}{2 \beta}}\|T u(t)\|_{\dot{B}_{p, \infty}^{w-(2 \beta-1)+\frac{n}{p}}\left(\mathbb{R}^{n}\right)} \\
\lesssim & \|a\|_{\dot{B}_{p, \infty}^{-(2 \beta-1)+\frac{n}{p}}\left(\mathbb{R}^{n}\right)} \\
& +t^{\frac{w}{2 \beta}} \int_{0}^{t}\left\|e^{-(t-s)(-\triangle)^{\beta}} P \nabla \cdot(u(s) \otimes u(s))\right\|_{\dot{B}_{p, \infty}^{w-(2 \beta-1)+\frac{n}{p}}\left(\mathbb{R}^{n}\right)} d s \\
\lesssim & \|a\|_{\dot{B}_{p, \infty}^{-(2 \beta-1)+\frac{n}{p}}}\left(\mathbb{R}^{n}\right) \\
\lesssim & \|a\|_{\dot{B}_{p, \infty}^{-(2 \beta-1)+\frac{n}{p}}}+\sup _{0<s<t} s^{\frac{w}{2 \beta}}\|(u(s) \otimes u(s))\|_{\dot{B}_{p, \infty}^{w-2 \beta+\frac{n}{p}}}\left(\sup _{0<s} s^{\frac{w}{2 \beta}}\|u(s)\|_{L^{\infty}\left(\mathbb{R}^{n}\right)}\|u(s)\|_{\dot{B}_{p, \infty}^{w-2 \beta+\frac{n}{p}}} s_{\left(\mathbb{R}^{n}\right)}\right. \\
\lesssim & \|a\|_{\dot{B}_{p, \infty}^{-(2 \beta-1)+\frac{n}{p}}}+\|u\|_{K}^{2}
\end{aligned}
$$

since $0<w-2 \beta+\frac{n}{p}<1, n \leq p<\infty$. Similarly, one has

$$
\|T u-T v\|_{K} \lesssim\left(\|u\|_{K}+\|v\|_{K}\right)\|u-v\|_{K}, \text { for } u, v \in K .
$$

These estimates imply that $T$ is a contraction mapping since $\|a\|_{\dot{B}_{p, \infty}^{-(2 \beta-1)+\frac{n}{p}}}\left(\mathbb{R}^{n}\right)$ is small enough. Therefore, we can finish the proof by the contraction mapping principle.

3.4. Proof of Theorem 1.5 We can write

$$
e^{-t(-\triangle)^{\beta}} f=e^{-(t-u)(-\triangle)^{\beta}} e^{-u(-\triangle)^{\beta}} f
$$

and

$$
e^{-t(-\triangle)^{\beta}} f(x)=\frac{2}{t} \int_{0}^{t / 2} e^{-(t-u)(-\triangle)^{\beta}} e^{-u(-\triangle)^{\beta}} f d s .
$$

According to the definition of $e^{-t(-\triangle)^{\beta}}$, it is a convolution operator with a positive Kernel

$$
K_{t}^{\beta}(x)=(2 \pi)^{-n / 2} \int_{\mathbb{R}^{n}} e^{i x \xi-t|\xi|^{2 \beta}} d \xi \quad \text { with } \quad K_{t}^{\beta}(x)=\frac{1}{t^{n / 2 \beta}} K_{1}^{\beta}\left(\frac{x}{t^{1 / 2 \beta}}\right) .
$$

Then, using Hölder's inequality, one has

$$
\begin{aligned}
\left|e^{-t(-\triangle)^{\beta}} f\left(x_{0}\right)\right| & =\left|\frac{2}{t} \int_{0}^{t / 2} \int_{\mathbb{R}^{n}} K_{t-u}^{\beta}\left(x-x_{0}\right) e^{-u(-\triangle)^{\beta}} f\left(x_{0}\right) d x d s\right| \\
& \lesssim \frac{2}{t} \int_{0}^{t / 2}\left(\int_{\mathbb{R}^{n}} K_{t-u}^{\beta}\left(x-x_{0}\right)\left|e^{-u(-\triangle)^{\beta}} f\left(x_{0}\right)\right|^{2} d x\right)^{1 / 2} \\
& \left(\int_{\mathbb{R}^{n}}\left|K_{t-u}^{\beta}\left(x-x_{0}\right)\right| d x\right)^{1 / 2} d s \\
& \lesssim \frac{2}{t} \int_{0}^{t / 2}\left(\int_{\mathbb{R}^{n}} K_{t-u}^{\beta}\left(x-x_{0}\right)\left|e^{-u(-\triangle)^{\beta}} f\left(x_{0}\right)\right|^{2} d x\right)^{1 / 2} d s \\
& \lesssim\left(\frac{2}{t^{\frac{\zeta-1}{\beta}}} \int_{0}^{t / 2} \int_{\mathbb{R}^{n}} K_{t-u}^{\beta}\left(x-x_{0}\right) u^{\frac{\zeta-1}{\beta}}\left|e^{-u(-\triangle)^{\beta}} f\left(x_{0}\right)\right|^{2} d x d s\right)^{1 / 2} .
\end{aligned}
$$


It follows from Miao, Yuan and Zhang's [17, Lemma 2.1] that

$$
K_{t-u}^{\beta}\left(x-x_{0}\right) \lesssim \frac{1}{(t-u)^{n / 2 \beta}} \frac{1}{\left(1+\frac{\left|x-x_{0}\right|}{(t-u)^{1 / 2 \beta}}\right)^{n+2 \beta}} .
$$

Thus

$$
\begin{aligned}
I & =\int_{\mathbb{R}^{n}} K_{t-u}^{\beta}\left(x-x_{0}\right) u^{\frac{\zeta-1}{\beta}}\left|e^{-u(-\triangle)^{\beta}} f(x)\right|^{2} d x \\
& \lesssim \int_{\mathbb{R}^{n}} \frac{1}{(t-u)^{n / 2 \beta}} \frac{u^{\frac{\zeta-1}{\beta}}}{\left(1+\frac{\left|x-x_{0}\right|}{(t-u)^{1 / 2 \beta}}\right)^{n+2 \beta}}\left|e^{-u(-\triangle)^{\beta}} f(x)\right|^{2} d x \\
& \lesssim \int_{\frac{x-x_{0}}{(t-u)^{\frac{1}{2 \beta}}} \in k+[0,1]^{n}} \frac{1}{(t-u)^{n / 2 \beta}} \frac{u^{\frac{\zeta-1}{\beta}}}{\left(1+\frac{\left|x-x_{0}\right|}{(t-u)^{1 / 2 \beta}}\right)^{n+2 \beta}}\left|e^{-u(-\triangle)^{\beta}} f(x)\right|^{2} d x
\end{aligned}
$$

Since $0<u<\frac{t}{2}$ and $\frac{t}{2}<t-u<t$,

$$
I \lesssim \frac{1}{t^{n / 2 \beta}} \int_{0}^{t / 2} \int_{\left|x-x_{0}\right| \leq t^{\frac{1}{2 \beta}}} u^{\frac{\zeta-1}{\beta}}\left|e^{-u(-\triangle)^{\beta}} f(x)\right|^{2} d x .
$$

This gives

$$
\begin{aligned}
& \left\|e^{-t^{2 \beta}(-\triangle)^{\beta}} f\right\|_{L^{\infty}\left(\mathbb{R}^{n}\right)} \\
\lesssim & \left(\frac{2}{t^{\frac{\zeta-1}{\beta}}} \frac{1}{t^{n}} \int_{0}^{t^{2 \beta}} \int_{\left|x-x_{0}\right| \leq t} s^{\frac{\zeta-1}{\beta}}\left|e^{-s(-\triangle)^{\beta}} f(x)\right|^{2} d x d s\right)^{1 / 2} \\
\lesssim & \left(\frac{2}{t^{\frac{\zeta-1}{\beta}}} t^{-n} \int_{0}^{t^{2 \beta}} \int_{\left|x-x_{0}\right| \leq t} s^{\frac{\zeta-1}{\beta}}\left|e^{-s(-\triangle)^{\beta}} f(x)\right|^{2} d x d s\right)^{1 / 2},
\end{aligned}
$$

that is,

$$
t^{\zeta}\left\|e^{-t^{2 \beta}(-\triangle)^{\beta}} f\right\|_{L^{\infty}\left(\mathbb{R}^{n}\right)} \lesssim\left(t^{-n} \int_{0}^{t^{2 \beta}} \int_{\left|x-x_{0}\right| \leq t} s^{\frac{\zeta-1}{\beta}}\left|e^{-s(-\triangle)^{\beta}} f(x)\right|^{2} d x d s\right)^{1 / 2}
$$

It follows from Miao, Yuan and Zhang's [17, Prorposition 2.1] that, for $s<0$, $f \in \dot{B}_{\infty, \infty}^{s}\left(\mathbb{R}^{n}\right)$ if and only if

$$
\sup _{r>0} r^{-s / 2 \beta}\left\|e^{-r(-\triangle)^{\beta}} f\right\|_{L^{\infty}\left(\mathbb{R}^{n}\right)}<\infty
$$

Thus, the previous estimate implies that $B M O^{-\zeta}\left(\mathbb{R}^{n}\right) \hookrightarrow \dot{B}_{\infty, \infty}^{-\zeta}\left(\mathbb{R}^{n}\right)$.

3.5. Proof of Theorem 1.6 We need the following lemma which can be proved easily.

LEMMA 3.1. For $\zeta \geq 0,(-\triangle)^{\zeta / 2} e^{-(-\triangle)^{\beta}}$ is a convolution operator with kernel $K^{\zeta, \beta}(x) \in L^{1}\left(\mathbb{R}^{n}\right)$.

We divide the proof into two parts. First, we prove that $f \in B M O^{-\zeta}\left(\mathbb{R}^{n}\right)$ under the assumption of the existence of a distribution $g \in B M O\left(\mathbb{R}^{n}\right)$ with $f=(-\triangle)^{\zeta / 2} g$. From this assumption, one has, for all $s>0$,

$$
s^{\zeta / \beta}\left|e^{-s(-\triangle)^{\beta}}(-\triangle)^{\frac{\zeta}{2}} g\right|^{2}=\left|K_{\zeta, s^{\frac{1}{2 \beta}}} * g\right|^{2}
$$


with

$$
K_{\zeta, s^{\frac{1}{2 \beta}}}(x)=s^{-\frac{n}{2 \beta}} K_{\zeta}\left(\frac{x}{s^{\frac{1}{2 \beta}}}\right)
$$

Here $K_{\zeta} \in L^{1}\left(\mathbb{R}^{n}\right)$ and

$$
\widehat{K_{\zeta, s^{\frac{1}{2 \beta}}}}(\xi)=\widehat{K_{\zeta}}\left(s^{\frac{1}{2 \beta}} \xi\right)=s^{\frac{\zeta}{2 \beta}}|\xi|^{\zeta} e^{-s|\xi|^{2 \beta}} .
$$

Thus

$$
\int_{\mathbb{R}^{n}} K_{\zeta}(x) d s=0 \text { and }\left|K_{\zeta}(x)\right| \lesssim \frac{1}{(1+|x|)^{n+\zeta}} .
$$

For more about the kernel of $e^{-t(-\triangle)^{\beta}}$, see Miao, Yuan and Zhang [17]. Then we have

$$
\begin{aligned}
\sup _{\xi \in \mathbb{R}^{n}} \int_{0}^{\infty}\left|\widehat{K_{\zeta}}(t \xi)\right|^{2} \frac{d t}{t} & =\sup _{|\xi|=1} \int_{0}^{\infty}\left|\widehat{K_{\zeta}}(t \xi)\right|^{2} \frac{d t}{t} \\
& =\sup _{|\xi|=1} \int_{0}^{\infty}\left(t^{\zeta} e^{-t^{2 \beta}}\right)^{2} \frac{d t}{t} \\
& =\int_{0}^{\infty} t^{2 \zeta-1} e^{-2 t^{2 \beta}} d t \\
& =\left(2^{-\frac{\zeta}{\beta}}-1\right) \beta^{-1} \Gamma(\zeta / \beta)<\infty
\end{aligned}
$$

since $\frac{\zeta}{\beta}>0$. So $d \mu(x, s)=\left|K_{\zeta, s^{\frac{1}{2 \beta}}} * g\right|^{2} \frac{d t d x}{s}$ is a Carleson measure and

$$
\iint_{0<s<t,\left|x-x_{0}\right|<t^{\frac{1}{2 \beta}}}\left|\left(K_{\zeta, s^{\frac{1}{2 \beta}}} * g\right)(x)\right|^{2} \frac{d s d x}{s} \lesssim\|g\|_{B M O\left(\mathbb{R}^{n}\right)}^{2} t^{\frac{n}{2 \beta}} .
$$

Thus $\|f\|_{B M O^{-\zeta\left(\mathbb{R}^{n}\right)}} \lesssim\|g\|_{B M O\left(\mathbb{R}^{n}\right)}$.

Second, we prove that there exists $g \in B M O\left(\mathbb{R}^{n}\right)$ with

$$
f=(-\triangle)^{\frac{\zeta}{2}} g \text { when } f \in B M O^{-\zeta}\left(\mathbb{R}^{n}\right) .
$$

Proposition 1.5 implies

$$
g=\sum_{j<0} g_{j}-g_{j}(0)+\sum_{j>0} g_{j}
$$

with $g_{j}=\triangle_{j} g$ such that $f=(-\triangle)^{\zeta / 2} g$ and $g \in \dot{B}_{\infty, \infty}^{0}\left(\mathbb{R}^{n}\right)$. In fact,

$$
\widehat{g}(\xi)=\sum_{j<0} \widehat{g_{j}}(\xi)-\widehat{g_{0}}(\xi)+\sum_{j>0} \widehat{g_{j}}(\xi)
$$

and

$$
\begin{aligned}
|\xi|^{\zeta} \widehat{g}(\xi) & =\sum_{j<0}|\xi|^{\zeta} \widehat{g_{j}}(\xi)-|\xi|^{\zeta} \widehat{g_{0}}(\xi)+\sum_{j>0}|\xi|^{\zeta} \widehat{g_{j}}(\xi) \\
& =|\xi|^{\zeta} \sum_{j \in \mathbb{Z}} \widehat{g_{j}}(\xi)=\sum_{j \in \mathbb{Z}} \widehat{\triangle_{j}}(f)(\xi)=\widehat{f}(\xi),
\end{aligned}
$$

according to the homogeneous Littlewood-Paley decomposition of $f$. On the other hand, to see $g \in \dot{B}_{\infty, \infty}^{0}\left(\mathbb{R}^{n}\right)$, we have,

$$
g_{j}=\triangle_{j} g=\triangle_{j}(-\triangle)^{\zeta / 2} f
$$


and

$$
\begin{aligned}
\widehat{g_{j}}(\xi) & =|\xi|^{-\zeta} \phi\left(2^{-j} \xi\right) \widehat{f}(\xi) \\
& =2^{-j \zeta}\left|2^{-j} \xi\right|^{-\zeta} \phi\left(2^{-j} \xi\right) \widehat{f}(\xi) \\
& =2^{-j r} h_{j}(\xi)\left|2^{-j} \xi\right|^{-\zeta} \phi\left(2^{-j} \xi\right) \widehat{f}(\xi) .
\end{aligned}
$$

Here $h_{j} \in C_{0}^{\infty}\left(\mathbb{R}^{n}\right)$ satisfying $h_{j}=1$ on $C_{j}$ and $\operatorname{supp}\left(h_{j}\right) \subset 2 C_{j}$. Let

$$
g_{j}=2^{-j \zeta} \triangle_{j} f *\left(h_{j}\left|2^{-j} \xi\right|^{-\zeta}\right)^{\vee},
$$

where $\left(h_{j}\left|2^{-j} \xi\right|^{-\zeta}\right)^{\vee} \in L^{\infty}\left(\mathbb{R}^{n}\right)$. It follows from $h_{j}\left|2^{-j} \xi\right|^{-\zeta} \in l^{\infty}(\mathbb{Z})$ that $\left\|\triangle_{j} g\right\|_{L^{\infty}\left(\mathbb{R}^{n}\right)} \in$ $l^{\infty}(\mathbb{Z})$. So

We need to prove that $g \in B M O\left(\mathbb{R}^{n}\right)$. In fact, let $\eta$ by $\widehat{\eta}\left(s^{\frac{1}{2 \beta}} \xi\right)=\left|s^{\frac{1}{2 \beta}} \xi\right|^{\zeta} e^{-s|\xi|^{2 \beta}}$.

and

$$
\widehat{\eta^{\frac{1}{2 \beta}} *} g(\xi)=\widehat{\eta}\left(s^{\frac{1}{2 \beta}} \xi\right) \widehat{g}(\xi)=\left|s^{\frac{1}{2 \beta}} \xi\right|^{\zeta} e^{-s|\xi|^{\beta}} \widehat{g}(\xi)
$$

$$
\widehat{g}(\xi)=\sum_{j \in \mathbb{Z}}|\xi|^{-\zeta} \widehat{\triangle}_{j}(f)(\xi)=|\xi|^{-\zeta} \widehat{f}(\xi) .
$$

This tells us

$$
\eta_{s^{\frac{1}{2 \beta}} *} g(\xi)=s^{\frac{\zeta}{2 \beta}} e^{-s|\xi|^{2 \beta}} \widehat{f}(\xi) \quad \text { and } \quad \eta_{s^{\frac{1}{2 \beta}}} * g(x)=s^{\frac{\zeta}{2 \beta}} e^{-s(-\triangle)^{\beta}} f(x) .
$$

Since $f \in B M O^{-\zeta}\left(\mathbb{R}^{n}\right)$ and $\eta$ satisfies the assumptions of Lemma 2.6,

$$
\begin{aligned}
& \left|B\left(x_{0}, t^{\frac{1}{2 \beta}}\right)\right|^{-1} \int_{0}^{t} \int_{\left|x-x_{0}\right|<t^{\frac{1}{2 \beta}}}\left|\eta_{s^{\frac{1}{2 \beta}}} * g\right|^{2} \frac{d s d x}{s} \\
= & t^{\frac{n}{2 \beta}} \int_{0<s<t} \int_{\left|x-x_{0}\right|<t^{\frac{1}{2 \beta}}}\left|s^{\frac{\zeta}{2 \beta}} e^{-s(-\triangle)^{\beta}} f(x)\right|^{2} \frac{d s d x}{s} \\
\lesssim & \sup _{t>0} \sup _{x_{0} \in \mathbb{R}^{n}}\left(t^{-n} \int_{0}^{t^{2 \beta}} \int_{\left|x-x_{0}\right|<t} s^{\frac{\zeta-1}{\beta \beta}}\left|e^{-s(-\triangle)^{\beta}} f(x)\right|^{2} d s d x\right) \\
\lesssim & \|f\|_{B M O-\zeta\left(\mathbb{R}^{n}\right)}^{2} .
\end{aligned}
$$

The previous estimate and Lemma 2.6 imply that $g \in B M O\left(\mathbb{R}^{n}\right)$. This finishes the proof of Proposition 1.6.

3.6. Proof of Proposition 1.9 The solution $(u, b)$ to equations (1.7) can be written as

with

$$
\begin{gathered}
u(t, x)=e^{-t(-\Delta)^{\beta}} u_{0}(x)-B(u, u)+B(u, u):=F_{1}(u, b), \\
b(t, x)=e^{-(-\triangle)^{\beta}} b_{0}(x)-B(u, b)+B(b, u):=F_{2}(u, b),
\end{gathered}
$$

$$
B(u, v)=\int_{0}^{t} e^{-(t-s)(-\triangle)^{\beta}} P \nabla \cdot(u \otimes v)(s) d s .
$$

Define

$$
Y=\left\{(u, b):(0, \infty) \longrightarrow G_{n}^{-(2 \beta-1)\left(\mathbb{R}^{n}\right)} \mid \nabla \cdot u=\nabla \cdot b=0,\|(u, b)\|_{Y}<\infty\right\}
$$

with

$$
\begin{gathered}
\|(u, b)\|_{Y}=\sup _{t>0}\left(\|(u, b)(t)\|_{G_{n}^{-(2 \beta-1)}\left(\mathbb{R}^{n}\right)}+t^{\frac{w-1}{2 \beta}}\|(u, b)(t)\|_{\dot{B}_{\infty, \infty}^{w-(2 \beta-1)}\left(\mathbb{R}^{n}\right)}\right)<\infty, \\
\|(u, b)\|_{Y}=\|u\|_{Y}+\|b\|_{Y} .
\end{gathered}
$$


We want to show that $F_{1}$ and $F_{2}$ are contraction mappings from a ball of $Y$ to itself. We rewrite the solution $(u, b)$ as

$$
\left(\begin{array}{c}
u \\
b
\end{array}\right)=\left(\begin{array}{c}
F_{1}(u, b) \\
F_{2}(u, b)
\end{array}\right):=F(u, b) .
$$

Then one has

$$
\begin{aligned}
& t^{\frac{w}{2 \beta}}\left\|F_{1}(u, b)(t)\right\|_{\dot{B}_{\infty, \infty}^{w-(2 \beta-1)}\left(\mathbb{R}^{n}\right)} \\
\lesssim & \left\|u_{0}\right\|_{\dot{B}_{\infty, \infty}^{-(2 \beta-1)}\left(\mathbb{R}^{n}\right)}+\sup _{0<s<t} s^{\frac{w}{2 \beta}}\|(u \otimes u, b \otimes b)(s)\|_{\dot{B}_{\infty, \infty}^{w-2 \beta}\left(\mathbb{R}^{n}\right)} \\
\lesssim & \left\|u_{0}\right\|_{\dot{B}_{\infty}^{-(2 \beta-1)}\left(\mathbb{R}^{n}\right)}+\sup _{0<s<t} s^{\frac{2 \beta-1}{2 \beta}}\|(u \otimes u, b \otimes b)(s)\|_{\dot{B}_{\infty, \infty}^{-(2 \beta-1)}\left(\mathbb{R}^{n}\right)} \\
& +\sup _{0<s<t} s^{\frac{w+2 \beta-1}{2 \beta}}\|(u \otimes u, b \otimes b)(s)\|_{\dot{B}_{\infty, \infty}^{w-(2 \beta-1)}\left(\mathbb{R}^{n}\right)} \\
\lesssim & \left\|u_{0}\right\|_{\dot{B}_{\infty, \infty}^{-(2 \beta-1)}\left(\mathbb{R}^{n}\right)}+\sup _{0<s<t} s^{\frac{2 \beta-1}{2 \beta}}\|(u \otimes u, b \otimes b)(s)\|_{G_{n}^{-(2 \beta-1)}\left(\mathbb{R}^{n}\right)} \\
& +\sup _{0<s<t} s^{\frac{w+2 \beta-1}{2 \beta}}\|(u, b)(s)\|_{\dot{B}_{\infty, \infty}^{w-(2 \beta-1)}\left(\mathbb{R}^{n}\right)}\|(u, b)(s)\|_{L^{\infty}\left(\mathbb{R}^{n}\right)} \\
\lesssim & \left\|u_{0}\right\|_{G_{n}^{-(2 \beta-1)}\left(\mathbb{R}^{n}\right)}+\sup _{0<s<t} s^{\frac{2 \beta-1}{2 \beta}}\|(u, b)(s)\|_{L^{\infty}}\|(u, b)(s)\|_{G_{n}^{-(2 \beta-1)}\left(\mathbb{R}^{n}\right)} \\
& +\sup _{0<s<t} s^{\frac{w+2 \beta-1}{2 \beta}}\|(u, b)(s)\|_{\dot{B}_{\infty, \infty}^{w-(2 \beta-1)}\left(\mathbb{R}^{n}\right)}\|(u, b)(s)\|_{L^{\infty}\left(\mathbb{R}^{n}\right)} \\
\lesssim & \left\|u_{0}\right\|_{G_{n}^{-(2 \beta-1)}\left(\mathbb{R}^{n}\right)}+\sup _{0<s<t}\left[s^{\frac{2 \beta-1}{2 \beta}}\|(u, b)(s)\|_{L^{\infty}}\right. \\
& \left.\left(\|(u, b)(s)\|_{G_{n}^{-(2 \beta-1)}\left(\mathbb{R}^{n}\right)}+s^{\frac{w}{2 \beta}}\|(u, b)(s)\|_{\dot{B}_{\infty, \infty}^{w-(2 \beta-1)}\left(\mathbb{R}^{n}\right)}\right)\right] \\
\lesssim & \left\|u_{0}\right\|_{G_{n}^{-(2 \beta-1)}\left(\mathbb{R}^{n}\right)}+\|(u, b)\|_{Y}^{2} .
\end{aligned}
$$

Similarly,

$$
\begin{aligned}
& \left\|F_{1}(u, b)(t)\right\|_{G_{n}^{-(2 \beta-1)}\left(\mathbb{R}^{n}\right)} \\
\lesssim & \sup _{s>0} s^{\frac{2 \beta-1}{2 \beta}}\left\|e^{-t(-\triangle)^{\beta}} e^{-s(-\triangle)^{\beta}}\left|u_{0}\right|(s)\right\|_{L^{\infty}\left(\mathbb{R}^{n}\right)} \\
& +\int_{0}^{t}\left\|e^{-(t-s)(-\triangle)^{\beta}} P \nabla \cdot(u \otimes u)(s)\right\|_{G_{n}^{-(2 \beta-1)}\left(\mathbb{R}^{n}\right)} d s \\
& +\int_{0}^{t}\left\|e^{-(t-s)(-\triangle)^{\beta}} P \nabla \cdot(b \otimes b)(s)\right\|_{G_{n}^{-(2 \beta-1)}\left(\mathbb{R}^{n}\right)} d s \\
\lesssim & \left\|u_{0}\right\|_{G_{n}^{-(2 \beta-1)}\left(\mathbb{R}^{n}\right)}+\int_{0}^{t}(t-s)^{\frac{2 \beta-1}{2 \beta}}\|(u, b)\|_{G_{n}^{-(2 \beta-1)}\left(\mathbb{R}^{n}\right)}\|(u, b)(s)\|_{L^{\infty}\left(\mathbb{R}^{n}\right)} d s \\
\lesssim & \left\|u_{0}\right\|_{G_{n}^{-(2 \beta-1)}\left(\mathbb{R}^{n}\right)}+\|(u, b)\|_{Y}^{2} .
\end{aligned}
$$

Thus, we get

$$
\left\|F_{1}(u, b)\right\|_{Y} \lesssim\left\|u_{0}\right\|_{G_{n}^{-(2 \beta-1)}\left(\mathbb{R}^{n}\right)}+\|(u, b)\|_{Y}^{2}
$$

and

$$
\left\|F_{1}(u, b)(t)-F_{1}\left(u^{\prime}, b^{\prime}\right)\right\|_{Y} \lesssim\left\|\left(u-u^{\prime}, b-b^{\prime}\right)\right\|_{Y}\left(\|(u, b)\|_{Y}+\left\|\left(u^{\prime}, b^{\prime}\right)\right\|_{Y}\right) .
$$


Similarly, we can prove that

$$
\left\|F_{2}(u, b)\right\|_{Y} \lesssim\left\|u_{0}\right\|_{G_{n}^{-(2 \beta-1)}\left(\mathbb{R}^{n}\right)}+\|u\|_{Y}\|b\|_{Y}
$$

and

$$
\left\|F_{2}(u, b)(t)-F_{2}\left(u^{\prime}, b^{\prime}\right)\right\|_{Y} \lesssim\left\|\left(u-u^{\prime}, b-b^{\prime}\right)\right\|_{Y}\left(\|(u, b)\|_{Y}+\left\|\left(u^{\prime}, b^{\prime}\right)\right\|_{Y}\right) .
$$

These estimates imply that

$$
\left\|F(u, b)-F\left(u^{\prime}, b^{\prime}\right)\right\|_{Y} \lesssim\left\|\left(u-u^{\prime}, b-b^{\prime}\right)\right\|_{Y}\left(\|(u, b)\|_{Y}+\left\|\left(u^{\prime}, b^{\prime}\right)\right\|_{Y}\right) .
$$

Therefore, the contraction mapping principle finishes the proof.

Acknowledgements. The author would like to thank Professor Jie Xiao for suggesting the problem and for all helpful discussions and kind encouragement.

\section{References}

[1] J. Bergh, J. Löfström, Interpolation Spaces: An Introduction, Springer, Heidelberg, 1976.

[2] J. Bourgain, N. Pavlović, Ill-posedness of the Navier-Stokes equations in a critical space in 3D, J. Funct. Anal. 255 (2008), 2233-2247.

[3] M. Cannone, Harmonic analysis tools for solving the incompressible Navier-Stokes equations, In: Handbook of Mathematical Fluid Dynamics Vol 3(eds. S. Friedlander, D. Serre), Elsevier, 2004, pp. 161-244.

[4] C. Cao, J. Wu, Two regularity criteria for the 3D MHD equations, J. Differential Equations (2009), doi:10.1016/j.jde.2009.09.020.

[5] Z. M. Chen, Z. Xin, Homogeneity criterion for the Navier-Stokes equations in the whole space, J. Math. Fluid Mech. 3 (2001), 152-182.

[6] G. Dafni, J. Xiao, Some new tent spaces and duality theorem for fractional Carleson measures and $Q_{\alpha}\left(\mathbb{R}^{n}\right)$, J. Funct. Anal. 208 (2004), 377-422.

[7] M. Essen, S. Janson, L. Peng, J. Xiao, Q space of several real variables, Indiana Univ. Math. J. 49 (2000), 575-615.

[8] M. Frazier, B. Jawerth and G. Weiss, Littlewood-Paley Theory and the Study of Function Spaces, CBMS Regional Conference Serices in Mathematics, 79, AMS, Providence (1991).

[9] Y. Giga, T. Miyakawa, Navier-Stokes flow in $\mathbb{R}^{3}$ with measures as initial vorticity and Morry spaces, Comm. Partial Differential Equtions 14 (1989), 577-618.

[10] L. Grafakos, Classical and Modern Fourier Analysis, Pearson (2004).

[11] H. Kozono, T. Ogawa, Y. Taniuchi, Navier-Stokes equations in the Besov space near $L^{\infty}$ and BMO, Kyushu J. Math. 57 (2003), 303-324.

[12] T. Kato, Strong $L^{p}$-solutions of the Navier-Stokes in $\mathbb{R}^{n}$ with applications to weak solutions, Math. Zeit. 187 (1984), 471-480.

[13] H. Koch, D. Tataru, Well-posedness for the Navier-Stokes equations, Adv. Math. 157 (2001), 22-35.

[14] P. Li, Z. Zhai, Well-posedness and regularity of generalized Navier-Stokes equations in some critical Q- spaces, arXiv:0904.3271v1 [math.AP], 2009.

[15] P. Li, Z. Zhai, Generalized Naiver-Stokes equations with initial data in local Q-type spaces, arXiv:0904.3283v1 [math.AP], 2009.

[16] J. L. Lions, Quelques méthodes de résolution des problèmes aux limites non linéaires, (French) Paris: Dunod/Gauthier-Villars, 1969.

[17] C. Miao, B. Yuan, B. Zhang, Well-posedness of the Cauchy problem for the fractional power dissipative equations, Nonlinear Anal. TMA 68 (2008), 461-484.

[18] H. Triebel, Theory of Function Spaces II, Birkhäuser, Basel, 1983.

[19] J. Wu, Generalized MHD euqations, J. Differential Equations 195 (2003), 284-312.

[20] J. Wu, The generalized incompressible Navier-Stokes equations in Besov spaces, Dynamics of PDE. 1 (2004), 381-400.

[21] J. Wu, Lower Bounds for an integral involving fractional Laplacians and the generalized Navier-Stokes equations in Besov spaces, Commun. Math. Phys. 263 (2005), 803-831.

[22] J. Wu, Regularity Criteria for the Generalized MHD Equations, Communications in Partial Differential Equations 33 (2008), 285-306. 
[23] J. Xiao, A sharp Sobolev trace inequality for the fractional-order derivatives, Bull. Sci. Math. 130 (2006), 87-96.

[24] J. Xiao, Homothetic variant of fractional Sobolev space with application to Navier-Stokes system, Dynamics of PDE. 2 (2007), 227-245.

[25] Z. Zhai, Global well-posedness for nonlocal fractional Keller-Segel systems in critical Besov spaces, Nonlinear Anal. TMA 72 (2010), 3173-3189.

[26] Y. Zhou, Regularity criteria for the generalized viscous MHD equations, Ann. Inst. H. Poincar Anal. Non Linaire 24 (2007), 491-505.

Department of Mathematical and Statistical Sciences, University of Alberta, 632 Central Academic Building Edmonton, AB T6G 2G1 Canada

E-mail address: zhichun1@ualberta.ca; a64zz@mun.ca 